\title{
Fabrication and Superconducting Property study of 1D Single Crystalline Metal Nanowires
}

\author{
Ying Zhang1, Xijun $\mathrm{Hu}^{2 \star}$ and Zifeng Yan ${ }^{1}$
}

'State Key Laboratory for Heavy Oil Processing, PetroChina Key Laboratory of Catalysis, China University of Petroleum, Qingdao 266580, China 2 DepartmentofChemical and Biomolecular Engineering, Hong Kong University of Science and Technology, Clear Water Bay, Kowloon, Hong Kong

\section{Article Info}

*Corresponding author:
Xijun Hu
Professor
Department of Chemical and Biomolecular
Engineering
Hong Kong University of Science and
Technology
Clear Water Bay, Kowloon
Hong Kong.
E-mail: kexhu@ust.hk

Received: October 3, 2016

Accepted: November 3, 2016

Published: November 8, 2016

Citation: Zhang Y, Hu X, Yan Z. Fabrication and Superconducting Property study of 1D Single Crystalline Metal Nanowires. Madridge J Nanotechnol Nanosci. 2016; 1(1): 29-43. doi: 10.18689/mjnn-1000108

Copyright: (c) 2016 The Author(s). This work is licensed under a Creative Commons Attribution 4.0 International License, which permits unrestricted use, distribution, and reproduction in any medium, provided the original work is properly cited.

Published by Madridge Publishers

\begin{abstract}
Physical properties of quasi-one-dimensional superconducting nanowires can differ significantly from those of bulk superconductors. The reason behind is the impact of thermal and quantum fluctuations. In the particular case of resistive measurements, the fluctuations may significantly broaden the superconducting transition $R(T)$. For superconducting wires with diameters decreasing, quantum phase slippage is an important process which can yield a non-vanishing wire resistance down to very low temperatures. Further decrease of the wire diameter results in proliferation of quantum phase slips causing a sharp crossover from superconducting to normal behaviour. The critical magnetic field exhibits quantumsize oscillations with pronounced resonant enhancements as a function of the wire radius. The understanding of the nature of superconductivity is often limited by the sample's morphology (e.g., disorder, defects, grain boundaries, or crystallinity). In order to identify the intrinsic phenomena in 1D, experimental studies of single crystal nanowires are clearly desirable. In this review, we present a summary of some recent activities in the field with main emphasis on experiment, while brief theoretical background is also presented.
\end{abstract}

Keywords: Fabrication; Superconducting nanowires; Magnetic field; Crystallinity.

\section{Introduction}

Advances in fabrication now allow the production of high-quality wires whose diameters are smaller than the superconducting coherence length. In this regime, transverse excitations in the superconducting order parameter are energetically less favourable, and the system approaches the $1 \mathrm{D}$ limit where the role of thermal fluctuations is more important than in 3D. Thus, superconductivity in quasi-one-dimensional (quasi-1D) nanowires is one of the most intriguing problems in condensed-matter physics [1-6]. Many interesting physical phenomena, such as thermally activated phase slip [7-9], quantum phase slip [1011], superconductor-insulator transition [12] and proximity effect [13-14], were observed in quasi-1D systems [15-16] Magnetic field lifts the time reversal symmetry of the spin and orbital states of paired electrons and suppresses the superconducting transition [17]; many interesting results such as magnetoresistance oscillations were also observed under a magneticfield [18-20].Sincethemostdetailed workin1Dortwo-dimensionalsuperconducting systems has been carried out in polycrystallineor amorphous samples, our understanding of the natureof superconductivity is often limited by the sample'smorphology (e.g., disorder, defects, grain boundaries, orcrystallinity). In order to identify the intrinsic phenomena in1D, performing experiments on a system having the least possible variations in morphology and microstructure could not only clarify the mechanisms of phenomena but also allow the exploration of new properties. Therefore, to understand these interesting behaviours, singlecrystal superconducting systems with controlled dimensionality are highly desirable. Yi et al [21] found the transition temperature in single crystal wires is close 
to thebulk $T_{C}$, but significantly suppressed in polycrystalline wires. Michotte et al [22] found superconductivity of $\mathrm{Pb}$ nanowires survives only in thicker polycrystalline wires (i.e., for diameters $.50 \mathrm{~nm}$ ) with single-crystalsegments, and resistance of wires of diameter below $50 \mathrm{~nm}$ increasewith decreasing temperature below $10 \mathrm{~K}$, with no evidenceof superconductivity due to "poor crystal structure". Tian et al [23]. found much more PSCs along the length in single crystal Sn wires than the polycrystalline wire might be due to single crystalline and polycrystalline morphology.These experiments further confirm the need ofmeasurement on quasi-1D single-crystal wires.Here we present an overview of recent results on superconducting nanowires in the quantum-size regime with main emphasis on experiment, while brief theoretical background is also presented.

\section{Superconducting metal nanowires $\mathrm{Nb}$ nanowires}

Since $\mathrm{Nb}$ is the most studied among the superconducting elements due to its high Tc $(9.5 \mathrm{~K})$ and the most used in superconducting electronics, it represents the most suitable material to test new possible applications of the superconductivity at the nanoscale.

Continuous $\mathrm{Nb}$ wires, 7-15 nm in diameter, have been fabricated by sputter-coating single fluorinated carbon nanotubes by Rogachev et al [24]. The resistance versus temperature, $R(T)$, as shown in Figure 1 (c) were well described by the Langer-Ambegaokar-McCumber-Halperin (LAMH) theory of thermally activated phase slips. Quantum phase slips were not observed. This possibly because the thicker polycrystalline $\mathrm{Nb}$ wires deposited on carbon nanotubes do not have weak links, and the grain boundaries seen in the TEM image (Figure 1a) do not produce tunneling barriers for supercurrent. It is quite surprising that the in homogeneities in granular $\mathrm{Nb}$ nanowires do not seemto broaden the $\mathrm{R}(\mathrm{T})$ curves, which can be fitted by the TAPS model developed forhomogeneous quasi-1D systems.

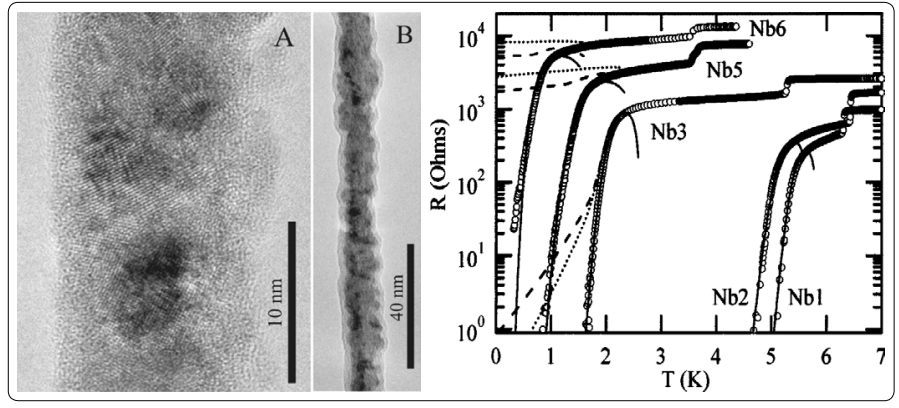

Figure 1(a). A high-resolution TEM image of a $\mathrm{Nb}$ nanowire fabricated by deposition of a $6 \mathrm{~nm} \mathrm{Nb}$ film over a single-wall carbon nanotube. (b)Image of one of the thinnest $\mathrm{Nb}$ nanowire (the thickness is $4 \mathrm{~nm}$ ) covered with a protective layer of Si (2 nm) visible

as light layer at the surface. (c)Temperature dependence of the resistance of superconducting $\mathrm{Nb}$ nanowires. Solid lines show the fits to the LAMH theory. The samples Nb1, Nb2, Nb3, Nb5, and Nb6 have the following fitting parameters. Transition temperatures $(\mathrm{K})$ are $T_{C}=5.8,5.6,2.7,2.5$, and $1.9 \mathrm{~K}$, respectively. Coherence lengths $(\mathrm{nm})$ are $\xi(0)=8.5,8.1,18,16$, and 16.5 , respectively. The dashed

lines are theoretical curves that include the contribution of quantum phase slips into the wire resistance with generic factors

$a=51$ and $B=51$. The dotted lines are computed with $a=51.3$ and $B=57.2$.
$\mathrm{Nb}$ layers with $\mathrm{d}_{\mathrm{Nb}}$ in the range 3-50 $\mathrm{nm}$ were deposited on CNT films by high-vacuum dc magnetron sputtering with a starting pressure in the low 10-8 mbar regime and in an $\mathrm{Ar}^{+}$ deposition pressur e of $4 \mathrm{mbar}[25]$.The main panel of Figure 2(a) showed a scanning electron microscope (SEM) micrograph of CNTNb3. As sketched in the inset, the dot-ended line in the figure gives $d_{\mathrm{CNT}}+2 \mathrm{~d}_{\mathrm{Nb}}$, where $\mathrm{d}_{\mathrm{CNT}}=9.5 \mathrm{~nm}$ and, for this sample, $\mathrm{d}_{\mathrm{Nb}}=$ $3 \mathrm{~nm}$. In Figure2 (b), decreasing $d_{N b}, T_{c}$ on remained finite, while full superconducting behavior was inhibited down to the lowest measured temperature $(T=250 \mathrm{mK})$ as observed for the samples CNTNb30 and CNTNb20. The sample with the thinnest Nb layer, CNTNb10, presented a semiconducting behavior without any trace of superconductivity. This suggested the presence of a superconductor-insulator (S-I) transition with decreasing $\mathrm{d}_{\mathrm{Nb}}$ with $R_{N} R Q\left(R_{N}\right.$ was the normal state resistance measured just before of the superconducting transition, $\mathrm{R}_{\mathrm{Q}}$ was the quantum resistance)as a crossover resistance. This result, observed in many low dimensional materials [26] as well as in SNWs [27,28], found many controversial explanations [26]. In the case of SNWs it is interpreted in terms of charg e localization [27], or as due to local magnetic moments pres ent in the wire [29]. Other mechanisms such as, for example, electron interactions with surface photons [30], invoked as possible cause of the reduction ofTc [31] and of the S-I transition [26] in low dimensional systems. In fact, being the $T_{c}$ on of the aggregates of SNWs and of the reference films the same, the electron-phonon (eph) coupling constant in these systems should also be the same. Therefore, the S-I transition should be ascribed to other causes. A more intriguing explanation concerns the nature of weak link constrictions (WLCs) presented inside a single polycrystalline SNW at the interfaces between the grains. In this case, the insulating or metallic nature of the WLCs determines if $R_{N}$ $>R_{Q}$ or $R_{N}<R_{Q}$ [25]. The hypothesis of the WLC at the base of the appearance of the $\mathrm{S}$-I transition seems to be the most appropriate due to the large number of interfaces present in CNTNb systems. In fact, for low values of $d_{N b \text {, }}$ the weak coupling between the interfaces WAS favored with the consequent development of the S-I transition.
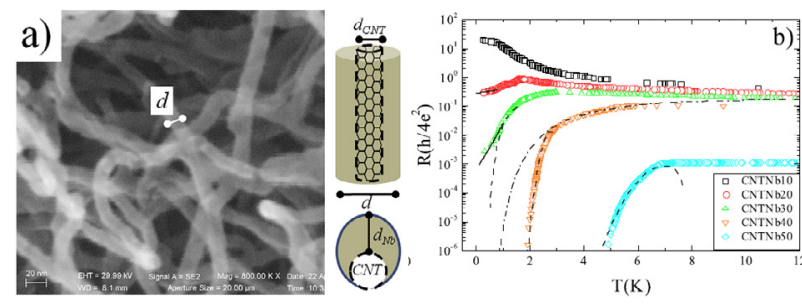

Figure 2(a). SEM image of the sample CNTNb3. (b)Resistance in units of quantum resistance as a function of T for the SNW aggregates. The lines in the right panel are fits to the data using the TAPS model (Eq. (1)-dashed lines), QPS model (Eq. (2)-solid lines) and Ambegaokar-Halperin (AH) model (dot-dashed line for CNTNb40). (A color version of this figure can be viewed online.)

Ultrathin nanowires of niobium, with diameters of $4.2 \mathrm{~nm}$ and perfectly clean surface, were grown in quantized vortices of superfluid helium [32]. The superconducting transition in the thin niobium nanowire was found to be distorted and strongly shifted towards lower temperatures. Superconducting niobium wires allowed a high rate of quantum phase slip. This phase slippage led to a phase transition to an insulating state 
at $T \rightarrow 0$ (shown in Figure 3(c)). This was in agreement with thetheory of quantum phase slips advanced by $Z$ aikin and co-workers [33] which predicts an insulating state at $T=0$. In this theory a superconducting to insulator transition critical point was expected to be controlled by the diameter of the nanowire. The critical diameter was estimated to be a few nanometers. The resistance is predicted to diverge in the insulating phase according to a power low, RT.
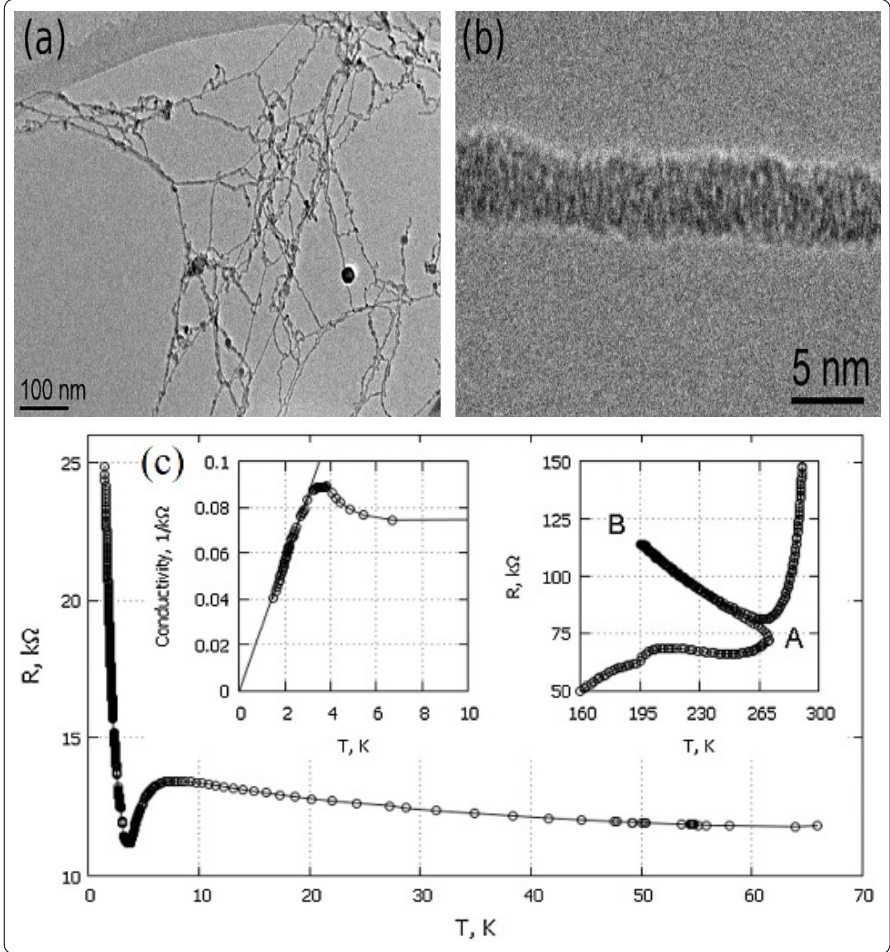

Figure 3 (a) and (b) Structure and morphology of the $\mathrm{Nb}$ nanowires. (c) The temperature dependence of the resistance of the bundle of niobium nanowires.

The $\mathrm{Nb}$ nanowires of $6 \mathrm{~mm}$ long with different crosssectional dimensions (shown in Figure $4 a, b$ ) were fabricated using standard e-beam lithography processes [34]. A lowtemperature residual resistance tail measured with an excitation current of $5 \mathrm{nA}$ was found in the thinnest wire down to $50 \mathrm{mK}$ or $7.7 \%$ of $\mathrm{Tc}$ of $\mathrm{Nb}$ (shown Figure $4 \mathrm{~d}, \mathrm{e}$ ). The functional form of the residual resistance was consistent with quantum phase slip (QPS) processes and was well described by eq 1 a s proposed by Giordano [11]. According to eq 1, authors defined $y=r /(1-T /$ $T)^{3 / 4}$ and $x=1$-, a plot of $\log (\mathrm{y})$ versus $\mathrm{x}$ in the low temperature regime should be a straight line if the measured resistance is dominated by QPS. The inset of Figure 4e confirmed this expectation. Moreover, resistance measured at high bias excitation current switched among many discrete values that were well below the normal state resistance. These discrete resistance values as a function of temperature falled into several parallel curves (shown Figure 5) all showing QPS-like decay in the low temperature limit similar to that found at low current. This phenomenon was not observed in the previous QPS experiments in micron long nanowires with either low or high currents and constitutes the key new result of Zhao's work. They quantified the length of the phase slip centers, which at about 40 $\mu \mathrm{m}$ was longer than the nanowires used in prior phase slip experiments but much shorter than their NbNW. The latter condition leaded to a situation that sections of the wire near phase slip centers turned normal while regions far from the phase slip centers stayed in superconducting phase and exhibited QPS-like temperature dependence in the low temperature limit similar to that found with low excitation current.

(1)
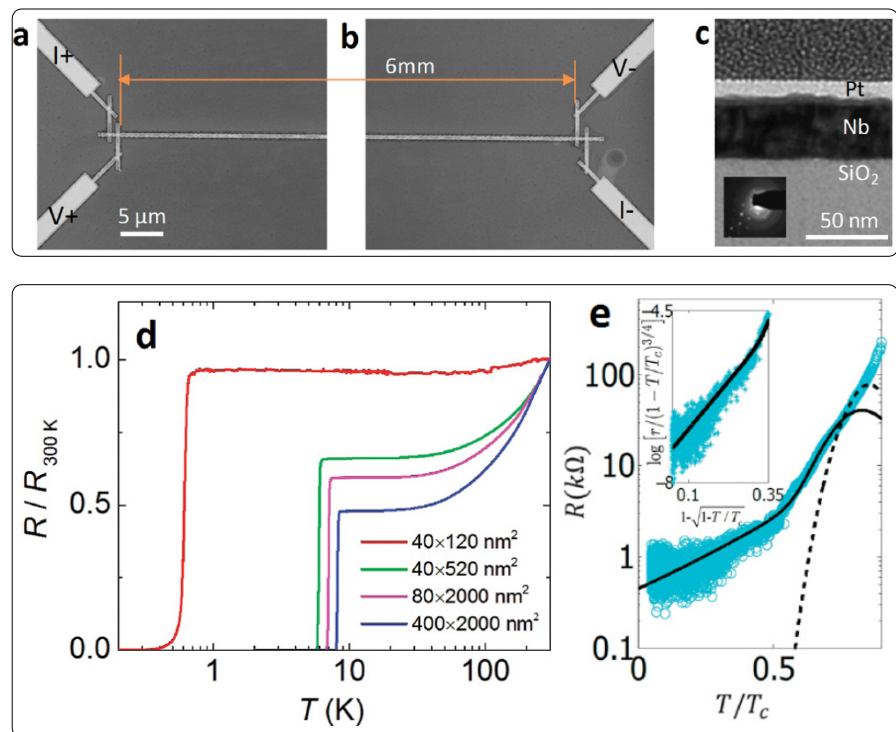

Figure 4 (a) and (b) SEM images of the two ends of a $6 \mathrm{~mm}$ long NbNW with width of $520 \mathrm{~nm}$ and the thickness of $40 \mathrm{~nm}$. (c) TEM image of the cross section of a $6 \mathrm{~mm}$ long NbNW of the same dimensions. The inset is an electron diffraction pattern for the NbNW.

(d) Resistance as a function of temperature $R(T)$ at zero field (normalized at $300 \mathrm{~K}$ ) for the $6 \mathrm{~mm}$ NbNWs with various cross-section dimensions. (e) The residual resistance tail shows QPS-like decay with temperature down to $50 \mathrm{mK}$ with excitation current of $5 \mathrm{nA}$.
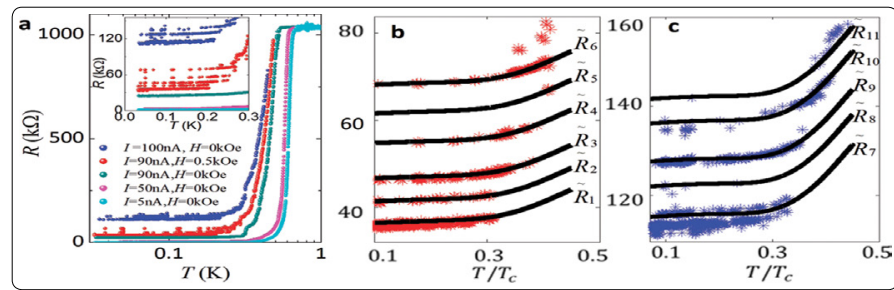

Figure 5(a). R-T scans at different I or $\mathrm{H}$ down to $50 \mathrm{mK}$ for the thinnest NbNW. The discrete values of $R(T)$ fall into several parallel curves exhibiting residual resistance expected of QPS. Red and blue stars are experiment data measured with $\mathrm{I}=90 \mathrm{nA}$ under $\mathrm{H}=0.5$ kOe (b) with I = $100 \mathrm{nA}$ und er $\mathrm{H}=0$ (c).

$X u$ et al [35] reported a general method, superlattice nanowire pattern transfer (SNAP), for reliably fabricating quasi-onedimensional superconducting nanowire arrays, with good control over nanowire cross section and length, and with full compatibility with device processing methods. The ability to fabricate very long (up to $100 \mu \mathrm{m}$ ) NWs with different lengths but identical cross sections has for the first time allowed for the investigation of length's sole influence on superconductivity in NWs, which are truly quasi1D superconductors at any temperature. Nearly atomically straight $\mathrm{Nb}$ nanowires with widths ranging from bulklike to $10 \mathrm{~nm}$ and aspect ratios approaching $10^{4}$ were prepared.Superconductivity was observed on all $\mathrm{Nb}$ nanowires arrays (Figure 6a).The superconductivity was partially suppressed (Tc $\sim 3.5 \mathrm{~K}$ as compared with $\mathrm{Tc} \sim 5 \mathrm{~K}$ for $\mathrm{Nb}$ contactedNWs of similar dimensions). The shorter NW array had a lower Tc due to higher suppression from the contacts. The thinner NWs had a lower Tc, with the cross-section dependence of Tc in general agreement with $d$ ata on CNTtemplated NWs [36]. Thinner Nb NW arrays $(11 \mathrm{~nm} \times 16 \mathrm{~nm} \times$

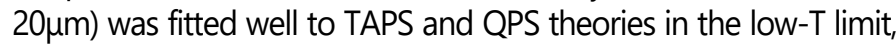


as shown in Figure 6b. The similar but shorter NWs deviated from QPS prediction. Such behaviour has been reported in short NWs [36-40], whereas long NWs typically show the expected QPS behaviour [41-43]. The authors concluded that QPS can be partially suppressed in short NWs in the low-T limit due to contact effects. Qualitatively different behaviours were also found for NWs with the same cross section but different lengths in Figure $6 \mathrm{~d}$ and e. In comparison with a single jump between the superconducting and normal states at Ic for shorter NWs (Figure 6d), longer NWs first enter an intermediate state at $\mathrm{lc}$, and jump to the normal state at a higher current level, $\mathrm{I}_{N} \sim 2 \mathrm{I}_{\mathrm{C}}$ (Figure 6e).This intermediate region exhibited multiple voltage jumps (Figure 6e inset). Such behaviour was characteristic of long quasi-1D superconductors, and each voltage jump was associated with the emergence of a localized resistive "phase-slip center" (PSC) along the wire. In contrast to the stochastic TAPS and QPS events, PSCs are stable and have fixed locations for a constant driving current. Each PSC occupies a characteristic length of $\sim 2 \Lambda_{\mathrm{Q}^{*}}$, where $\Lambda_{\mathrm{Q}^{*}} \sim 10 \mu \mathrm{m}$ is the quasiparticle diffusion length. The number of PSCs a wire can accommodate should be $\sim \mathrm{L} / 2 \Lambda_{\mathrm{Q}^{*}}$. But the results on NWs, which were truly quasi1D superconductors at any temperature, have been limited. For example, in electrodeposited Sn NW arrays, PSCs were pinned down at local defects, and the number of PSCs observed was independent of the length of the arrays [23].
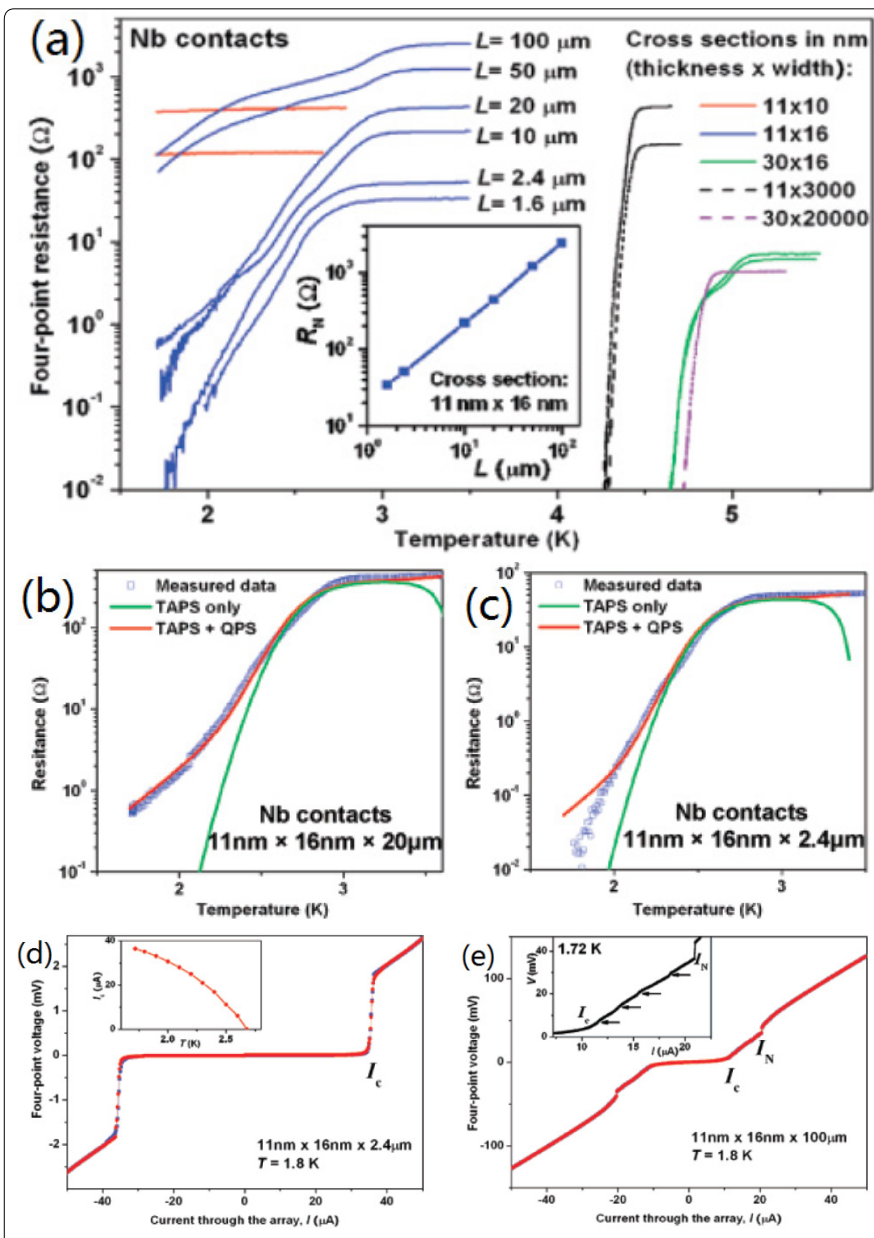

Figure 6. Temperature dependence for the four-point resistance of $\mathrm{Nb}$ NW arrays (a) Superconducting Nb contacted NW arrays and films.(b) $11 \mathrm{~nm} \times 16 \mathrm{~nm} \times 20 \mu \mathrm{m}$ data fitted to the theories.(c) $11 \mathrm{~nm} \times 16 \mathrm{~nm}$ $\times 2.4 \mu \mathrm{m}$ data fitted to the theories. Four-point V-I curves for (d) An array of $100 \mathrm{NWs}$ of cross section $11 \mathrm{~nm} \times 16 \mathrm{~nm}$ and $\mathrm{L}=2.4 \mu \mathrm{m}$. Inset: temperature dependence of Ic. (e) An array of $100 \mathrm{NWs}$ of cross section $11 \mathrm{~nm} \times 16 \mathrm{~nm}$ and $\mathrm{L}=100 \mu \mathrm{m}$. Inset: Expanded plot of the intermediate-state region (Ic $<\mathrm{I}<\mathrm{IN}$ ); arrows point to voltage jumps.

\section{$\mathrm{Pb}$ nanowires}

Bulk $\mathrm{Pb}$ is a well-known elementary superconductor with $83 \mathrm{~nm}$ coherence length. Therefore, $\mathrm{Pb}$ nanowires thinner than $\sim 80 \mathrm{~nm}$ are potential candidates to study the complex quasi-1D superconducting transition and associated finite size effects. Low dimensional $\mathrm{Pb}$ nanostructures have been ext ensively studied for decades [19,44-49]. Additionally, amorphous and granular nanowires of $\mathrm{Pb}$ and other materials ha ve been studied systematically $[1,15,19]$. In the last few years, there are a number of experiments studying the properties of single crystal superconducting nanowires with diameter less than $100 \mathrm{~nm}$ [23]. An overarching theme of these studies is to understand how superconductivity in these wires goes away with decreasing diameter.

Lead nanowires with high aspect ratio and diameter from 40 to $270 \mathrm{~nm}$ have been grown by electrodeposition inside nanoporous polycarbonate membranes [50]. The thinner $\mathrm{Pb}$ nanowires were found to be polycrystal with good quality single-crystal regions extending over several microns(asshown in Figure7a and $b$ ), and Figure $7 c$ and $d$ revealed the $\mathrm{Pb}$ nanowires with $95 \mathrm{~nm}$ in diameter appear to have a monocrystalline fcc structure. For small nanowires diamet er (56 nm), Ginzburg-Landau theory [51] allowed one to deduce that vortices did not penetrate nanowires. In contrast, for large nanowire diameter $(270 \mathrm{~nm})$, it was shown that one vortex likely penetrated the nanowires at low temperature.
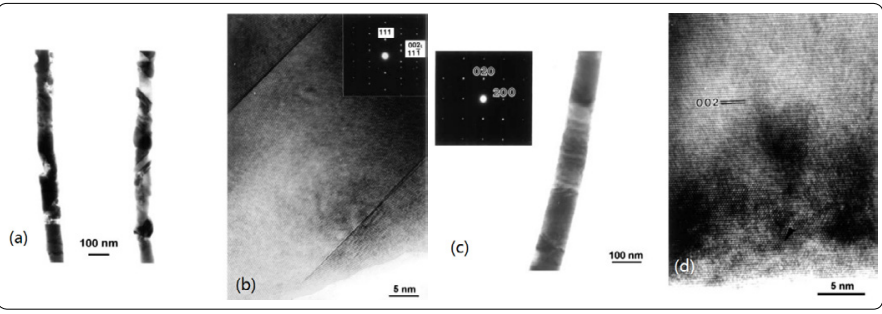

Figure 7. Transmission electron micrographs of lead nanowires showing their shape and their fine structure. (a) defects in $70 \mathrm{~nm}$ wires; (b ) high-resolution image and SAD pattern along a [110] zone axis of a (111) twin in a $70 \mathrm{~nm}$ wire; (c) Straight-sided $95 \mathrm{~nm}$ wire and

the corresponding SAD pattern along a [002] zone axis; (d) highresolution image showing the edge and the ce nter of a $95 \mathrm{~nm}$ wire.

Michotte et al [52], [22] reported transport properties of single lead granular nanowires grown by an electrodeposition technique with uniform diameter of $\sim 40 \mathrm{~nm}$ and a very large aspect ratio $(\sim 500)$. Due to the granular character of the nanowire, phase fluctuations were dominant and gave a non-zeroresistance presenting different stages in the superconducting state which caused by the formation of phase slip centers. Moreover, DC voltage driven measurements revealed the existence of a new S-shape behavior near the formation of these phase slip centers, as shown in Figure8. A theoretical analysis b ased on the generalized TDGL equations [53] gives a qualitative explanation of this phenomenon which is driven by a highly nonlinear time response of the superconductor to electric fields leading to the periodic creation of PS centers. Experiments with individual $\mathrm{Pb}$ nanowires showed clear superconducting transitions,which got broadened with red uction of the sample diameter (Figure 8b [54]. Unfortunatelyno theory fits was provided by the authors, while the 
linear scale of thereported $R(T)$ dependencies complicates an independent quantitative comparison.
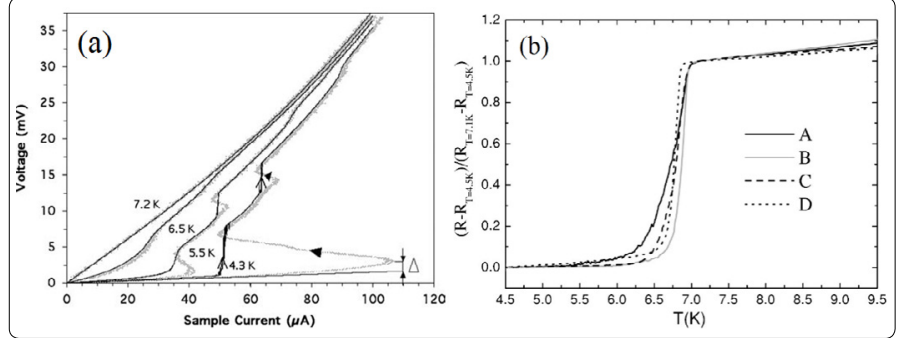

Figure 8. (a)Voltage-current characteristics at different temperatures of $\mathrm{Pb}$ nanowires ((diameter $40 \mathrm{~nm}$, length $22 \mu \mathrm{m})$ ). (b) R.T / dependence for four Pb nanowires A - $\mathrm{D}$ with diameters 40,

55,55 , and $70 \mathrm{~nm}$,respectively. The transition is broader for narrower wires.

Another set of quenchcondensedgranular $\mathrm{Pb}$ nanowires with cross-section areao down to $15 \mathrm{~nm}^{2}$ and lengthL ranging from 1 to $2 \mu \mathrm{m}$ was performed by Dynes and co-workers [12]. The samples edge roughness was claimed to be about $3 \mathrm{~nm}$. A remarkable feature of the method is the ability to vary the wire thicknessand, hence, its cross-section at a constant width $w$ in situ inside the cryostat inbetweenthe sessions of truly 4-probe $R(T)$ measurements. The experiments clearlyindicated systematic deviations of the experimental data points $R(T)$ from theTAPS model predictions. This discrepancy increases as the wires becomenarrower. The width of the superconducting transition was found to scale with thenormal state resistance $R_{N}$. It should be noted that in lead nanowires as narrowas $15 \mathrm{~nm}$ and as thin as $10 \mathrm{~nm}$ no low temperature resistance tails were observed. Instead, a less dramatic but systematic broadening of the superconducting transitionbeyond the TAPS limit was noted.

Single crystal superconducting $\mathrm{Pb}$ wires (diameter 50 nm) (Figure 9a) prepared by pulse electrodeposition innanoporous membranes [21] showed significantly higher superconducting transition temperature Tc than that of polycrystalline ones (Figure 9b). The author attributed this to the possible reasons:

(i) homogeneous disorder, which was reasonable, given that the grain boundary separation in the polycrystalline $\mathrm{Pb}$ nanowires wass less than the coherence length $\xi_{0}$ for $(\mathrm{Pb} \sim 83$ $\mathrm{nm})$;

(ii) The thermal conductivityof the polycrystalline nanowires was so low comparedto that of the single-crystal ones that their Tc appearssuppressed.
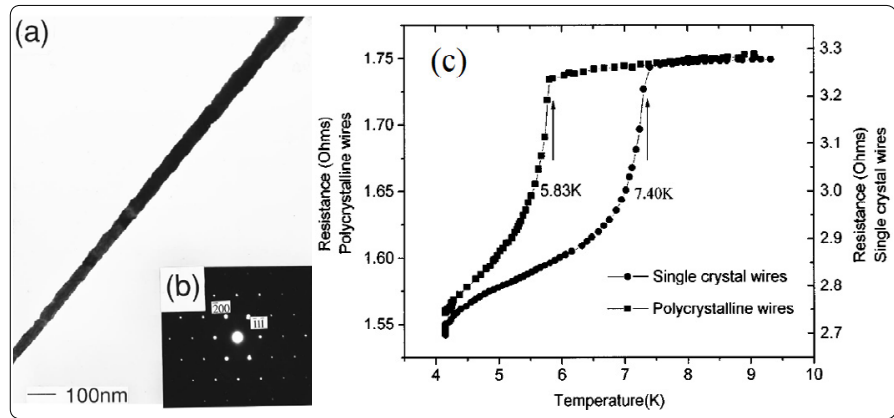

Figure 9 (a)TEM image of a single-crystal Pb nanowire. (b) $R(T)$ curves of for polycrystalline and sing le-crystal $\mathrm{Pb}$ nanowire samples.
He et al [55] fabricated ultrathin lead $(\mathrm{Pb})$ nanowires in the 6 $\mathrm{nm}$ pores of SBA-15 mesoporous silica substrates by chemical vapor deposition.The quasi-1D nature of the material causes obviously a more continuous transition in contrast to the sudden occurrence of the Meissner effect in bulk $\mathrm{Pb}$, as shown in Figure 10a. shows an enlarged transition onset. A tiny but sharp downturn in the ZFC magnetization at $11 \mathrm{~K}$ resolved in Figure $10 \mathrm{~b}$ suggested that the nanostructure induces weak diamagnetic fluctuations, 3 4 $\mathrm{K}$ above the bulk Tc. Tc enhancements in nanomaterial have been previously observed [56-59], but in this ultrathin $\mathrm{Pb}$ nanowires the effect was particularly strong. The surface of the thin nanowires represents a significant fracti on of the total volume, and phonon softening [60] can cause a locally higher Tc. As seen in Figure 10d, magnetic fields suppress the transition until the normal state was restored in fields slightly higher than $15 \mathrm{~T}$. This high upper critical field was further confirmed by the magnetoresistance data in the inset Figure 10e, which extrapolate to a field-independent value in fields slightly higher than $15 \mathrm{~T}$. The high upper critical field was attributed tothe replacement of the orbital limit for superconductivity by the Pauli limit in this quasi-1D superc onducting composite material on the nanoscale [61-64].
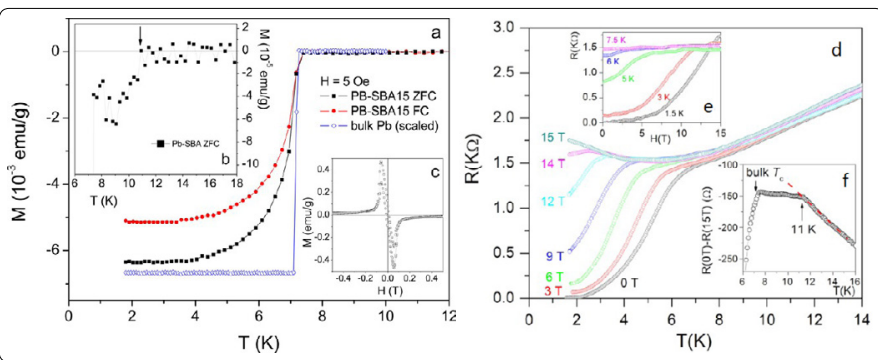

Figure 10 (a). Meissner signal in the magnetization of Pb-SBA-15. (b) Magnification of the onset of the superconducting transition of Pb-SBA-15 under ZFC conditions. (c) Field-sweep data for Pb-SBA-15 measured at $\mathrm{T}=1.8 \mathrm{~K}$.(d) Electrical resistance of Pb-SBA-15. (e) Magnetoresistance at varioustemperatures. (f) Enlarged view of the onset of the transition in zero fie ld after subtraction of $15 \mathrm{~T}$ data.

Wang et al [65] produced single-crystal $\mathrm{Pb}$ nanobelts of 28 atomic monolayers thick, $285 \mathrm{~nm}$ wide, and $10 \mu \mathrm{m}$ long by focus ion beam technique. In Figure11a, it shows that the onset TC $(6.9 \mathrm{~K})$ of the $\mathrm{Pb}$ nanobelt is obviously higher than that (6.3K) of the $\mathrm{Pb}$ film. Figure $10 \mathrm{~b}$ shows magnetoresistance oscillations at various temperatures below TC in both positive and negative perpendicular fields. This figure gives evidence that the oscillation in the $\mathrm{Pb}$ nanobelt is reproducible (not just some sort of noise) and might be considered quasiperiodic.
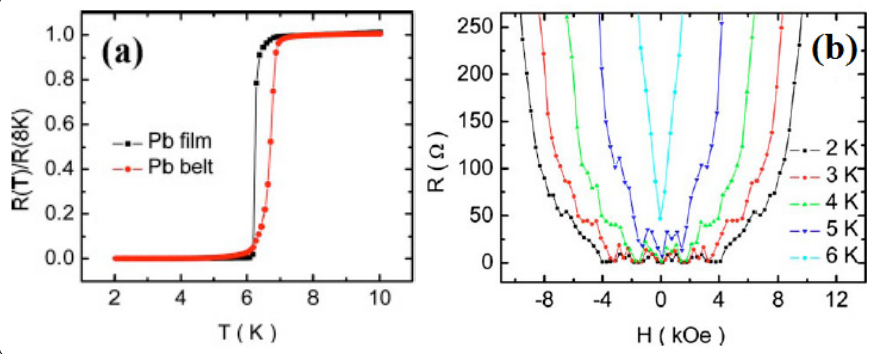

Figure 11 (a). Resistance as a function of temperature measured from the $\mathrm{Pb}$ film i and the $\mathrm{Pb}$ nanobelt (b)Magnetoresistance oscillations of the $\mathrm{Pb}$ nanobelt at 2, 3, 4, 5 and $6 \mathrm{~K}$, respectively, with both positive and negative perpendicular fields. 
It was found for individual thicker $\mathrm{Pb}$ nanowires that the material of the electrodes can have a strong influence on the superconducting state, even at distances between the voltage terminals exceeding the superconducting coherence length. Wang et al [65] fabricated single crystal Pb nanowires with two different diameters (55 nm and $70 \mathrm{~nm}$ ) by electrochemical deposition. R-T, R-H and R-I curves measured by standard fourprobe configuration showed a series of resistance steps with increasing temperature, magnetic field, and excitation current respectively as the superconducting nanowires were approaching the normal state. They attributed these steps to PSCs in the Pb nanowires enhanced by the proximity to the normal Pt electrodes.

\section{Sn nanowires}

Bulk tinhas a relatively long coherence length of $\xi_{0} \approx 200 \mathrm{~nm}$. Up to now, studies of superconducting properties of metallic Tinnanowires have revealed very interesting behaviour associated with their reduced physical dimension [66-68]. The onset critical field $(\mathrm{Hc})$ and transition temperature $(\mathrm{Tc})$ can be significantly affected bythe diameter and crystal textures of the nanowires.

Tian et al. investigated electrical transport properties of the single-crystal tin nanowires with diameters of $40-160 \mathrm{~nm}$ [66]. The resistance data for all single-crystal wires showed an onset of superconducting transition near $3.7 \mathrm{~K}$, the bulk $\mathrm{T}_{\mathrm{C}}$ of tin. The resistance drop for $40 \mathrm{~nm}$ exhibited two distinct regimes below $\mathrm{T}_{\mathrm{C}}$ one between 1.7 and $3 \mathrm{~K}$, and another between 3 and $3.7 \mathrm{~K}$, which may be due to a superposition of mally activated phase-slip resistance near $T_{C}$ [67], plus quantum phase-slip (QPS) far below $\mathrm{T}_{C}$ [68]. The $\mathrm{h}_{\mathrm{c}}$ for $40 \mathrm{~nm}$ was found to be $\sim 0.3 \mathrm{~T}$ at $1.8 \mathrm{~K}$, an order of magnitude higher than that of bulk $\mathrm{Sn}$. These results $w$ ere consistent with the observation of Dubois et al [69] and Michotte et al [52] in Pb wires. However, the $M(T)$ and $M(H)$ curves in single-crystal tin wires did not show their reversibility or hysteretic behaviour observed in the $\mathrm{Pb}$ wires Figure 12A. The reversible $M(H)$ curves of tin wires indicates a lack of pinning of residual flux in contract to the interpretation of in $\mathrm{Pb}$ wires [70-71].
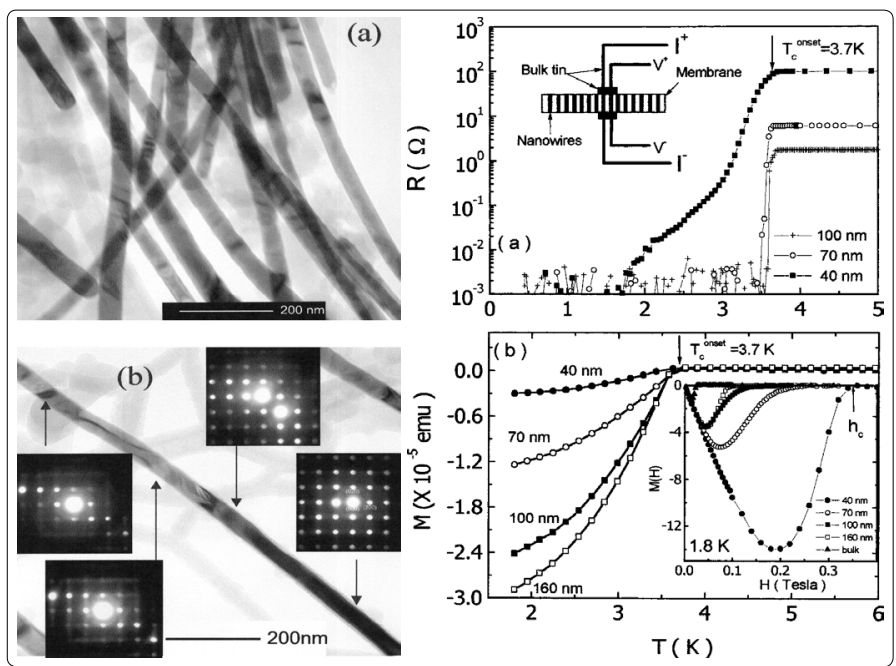

Figure 12A (a).TEM image of freestanding 40-nm Sn nanowires. (b) TEM image of a selected $40 \mathrm{~nm}$ Sn nanowire and its ED patterns along the wire length. (c) R-T curves of 40, 70 and $100 \mathrm{~nm}$ Sn nanowire array; the inset shows the schematic of the measurement arrangement. (d) M-T curves of 40,70, 100 and $160 \mathrm{~nm} \mathrm{Sn}$ nanowire array measured at 0.01-T magnetic field along the wire axis. The inset shows the $M(H)$

curves of the $\mathrm{Sn}$ nanowire array with various diameters at $1.8 \mathrm{~K}$.
Jankovič et al. fabricated carbon nanotubes encapsulating single crystalline tin nanowires with diameters $15-35 \mathrm{~nm}$ by empl oying the catalytic chemical vapour deposition method [72]. The magnetization measurements of Sn-CNT showed a small increase of the Tc (3.72K) onset because of the thinnest nanowires in which the confinement and surface-to-volume ratio becomes large enough to influence the vibrational and electronic properties. The same has been reported in the literature [23] for tin nanowires of $20 \mathrm{~nm}$ diameter. Moreover, the Sn-CNT showed a considerable increase (more than 30 times) of the critical magnetic field $\left(\mathrm{H}_{\mathrm{c}} 0.6\right.$ $\mathrm{T})$ compared to bulk metallic tin $(0.021 \mathrm{~T})$. The ultrapure tin nanowires are in the clean limit of the microscopic BCS theory, where the mean free path, $l$, is longer than the coherence length $\boldsymbol{\xi}(\boldsymbol{l \xi})$. In such a case, a nanocylinder of radius $\mathrm{R}$ smaller than the coherence length $R<\xi$ and with a magnetic field applied along its axis has a critical field $H_{c}=\exp (3 / 2) \Phi_{0} / \pi 2 \xi R$, where $\Phi_{0}=h / 2 e$ is the quantum of flux. Thus, the thinnest wires have a strongly enhanced critical field [73-75].
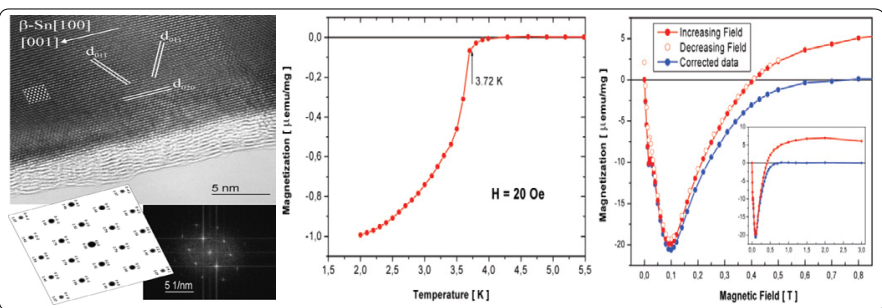

Figure 12B (a) High-resolution TEM image showing the high degree of crystallinity of both the MWCNTs and the metallic tin nanowires.

(b) Magnetization versus temperature curve, taken at 20 Oe. (c) Magnetization versus magnetic field curve, taken at $2 \mathrm{~K}$.

A noncatalytic and template-free vapour transport process has been employed to prepare $s$ ingle-crystalline Sn nanowires with diameters of 10-20nm [73]. The superconducting transition temperatures for $\mathrm{Sn}$ nanowires was about $3.7 \mathrm{~K}$, which was very close to that of bulk $\beta$-Sn. Magnetization measurements showed that the critical magnetic fields for both Sn nanowires increased significantly as compared to that of bulk Sn Figure 12B.

Tian et al. were fabricated $\mathrm{Sn}$ nanowires by electrodepositing tin into a porous membrane at room temperature [23]. The wires diameters was 20,40,60,70, and 100nm and the length was about $6 \mu \mathrm{m}$. The onset temperature Tc of nanowires was found to be close to the bulk value at $3.7 \mathrm{~K}$ for all wires with $\mathrm{d} \geqslant 40 \mathrm{~nm}$. A slight increase in $\mathrm{Tc}$ to $4.1 \mathrm{~K}$ was found for the thinnest, i.e., $20 \mathrm{~nm}$ wire. One possible origin was that this was an effect of the increased surface area, wh ich may enhance surface electron-phonon scattering effects [75]. In wires of 40 and $20 \mathrm{~nm}$, a clear functional dependence of the residual resistance on temperature was found .Th e broadening of the transition near Tc in thin whiskers [76-77] and In nanowires has been o bserved and was interpreted as a consequence of a TAPS process $[10,78]$. The low-temperature exponential residual resistance that extends over a wide range of temperature down to $0.47 \mathrm{~K}$ could not be understood in the framework of the TAPS model. A similar exponential de cay in resistance down to low temperature was found by Giordano [79] in granular indium wire of $41 \mathrm{~nm}$ in diameter, fabricated from evaporated In film with step-edge lithographic technique. Giordano proposed a phenomenological QPS model to explain his results. The finite exponentially decaying in resistance is 
proposed to result from quantum fluctuation-induced tunnelling through an energy barrier $\Delta \mathrm{F}$, resulting in a resistance $\mathrm{R}_{\mathrm{QPS}}$ of the

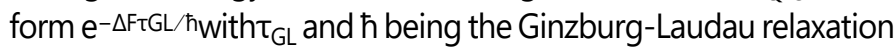
time and Planck's constant, respectively. The V-I curves of Sn thinner wires demonstrated that the finite resistance measured at the low current limit $(1<2 \mu \mathrm{A})$ was ohmic in nature at all temperatures, which lends further credence to the phenomenological QPS model with a tunnelling energy barrier. The V-I curves for 20 and $40 \mathrm{~nm}$ wires showed a series of voltage steps in approaching the normal state over the entire temperature range of $\mathrm{T}<\mathrm{Tc}$. The $\mathrm{V}-\mathrm{I}$ characteristics of the $\mathrm{Sn}$ wires exhibited features were similar to the experiments of Michotte et al. and consistent with their proposed model. Michotte et al [54] investigated the V-I characteristics of polycrystalline Sn nanowires with $50 \mu \mathrm{m}$ in length and $55 \mathrm{~nm}$ in diameter fabricated by electrodeposition method. Their results showed two voltage steps in the V-I curve under constant current-driving mode. Michotte et al [52] proposed a model using the generalized time-dependent Ginzburg-Landau equation. The theory indicated that the competition between two relaxation times (relaxation time of the absolute value of the order parameter $\tau|\psi|$ and relaxation time of the phase of the parameter $\tau|\phi|$ in the PSC) governs the phase slip process (i.e., $\tau|\phi|<\tau|\psi|$ ). They discussed the effect of magnetic field and defects on both relaxation times and V-I curve. An important conclusion of their model is that under a magnetic field that exceeds a critical value $\left(H^{\star}\right)$, the voltage jumps (PSCs) disappear in the V-I curve. The reason is that the magnetic field suppresses the order parameter $|\psi|$ everywhere in the sample, leading to an increase of $\wedge q \sim 1 / V(|\psi|)$, and hence there is a lack of space for the coexistence of two PSCs in the wire. The model also predicts that there will be no hysteresis near the voltage steps when the defects in the wire are sufficiently "strong." However, Figures $13 \mathrm{c}$ and d showed at least six voltage steps (PSCs) in individual 40nm Sn wire of $6 \mu \mathrm{m}$ in length, and much more PSCs along the length in $20 \mathrm{~nm} \mathrm{Sn}$ wires than the single 40nm wire. The different results between Tian et al. and Michotte et al. might be single crystalline and polycrystalline morphology.

Zhang et al. synthesized single crystalline Sn nanowires with typical thickness of $60-70 \mathrm{~nm}$ in diameter and with average length of $500 \mathrm{n} \mathrm{m}$ using surfactant as soft template by a chemical reduction process [80] (Figure 14a). The resistivity data of $\mathrm{Sn}$ nanowires showed a strong enhancement of the onset Tc and a high upper critical field(Figure 14b). Herzog et al [1] have reported Tc onsets of much thicker granular $\mathrm{Sn}$ nanowires somewhat above $4 \mathrm{~K}$ which may have been caused by the granularity. A slight increase in Tc to $4.1 \mathrm{~K}$ was also found for the $20 \mathrm{~nm} \mathrm{Sn}$ wire po ssible dueto the enhanced surface electron-phonon scattering effects [23]. the large width of the superconducting transition observed in the specific heat was at tributed to a large distribution of different coupling strengths $[81,82]$. The additional transition in the specific heat at $5.5 \mathrm{~K}$ was related to an enhanced effectiv e electron-phonon interaction near the surface of a superconductor [55, 83]. In addition, the curvature of the surface may represent another i mportant ingredient for the Tc enhancement in these cylindrical nanowires [84]. Without these effects, the Tc of a superconductor would rather be expected to decrease with dimensionality [85-86]. The critical field of this Sn nanowires (3T) was enhanced by about two orders of magnitude with respect to $\mathrm{Sn}$ in its bulk for $\mathrm{m}(0$. $03 \mathrm{~T})$, possibly because of the particularly strong spin orbital coupling [87-88] in a metallic surface state band.
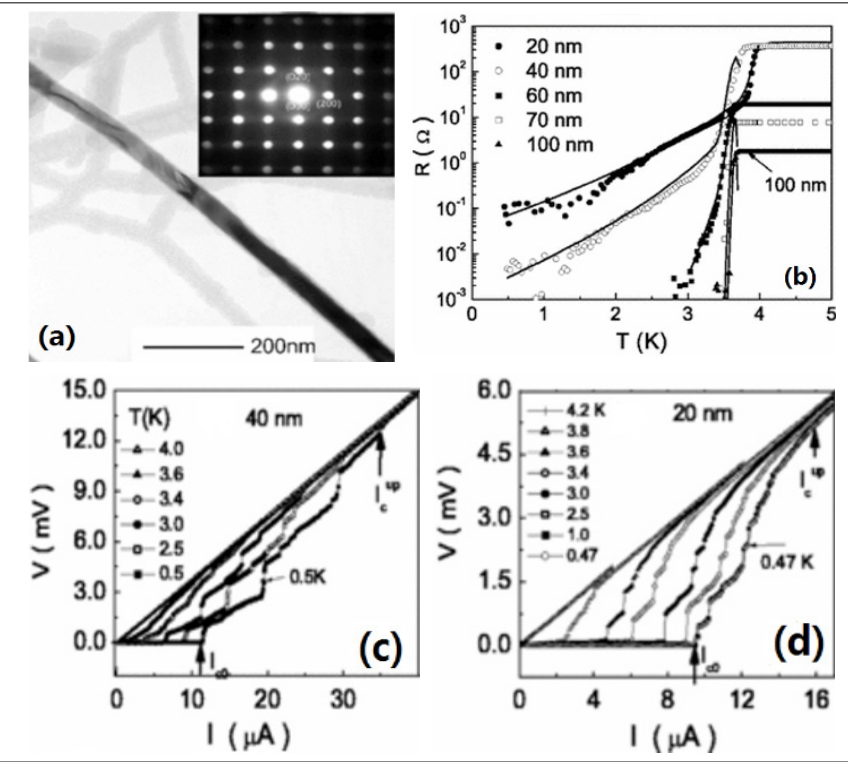

Figure 13 (a) The TEM image and the electron diffraction pattern of the individual 40nm wire. (b)R.T / curves for 20, 40, 60, 70, and 100 $\mathrm{nm}$ wide and $6 \mu \mathrm{m}$ long $\mathrm{Sn}$ nanowire arrayscontaining, respectively,

$18,1,8,15$ and 53 wires in the bundle. The solid lines for 20, 40, and60 $\mathrm{nm}$ wires are the results based on the TAPS model near $T_{C}$ and QPS model below $T_{C}$. (c) and (d) V-I curves of 40 and $20 \mathrm{~nm} \mathrm{Sn}$ nanowire arrays measured at different temperatures in linear scale.
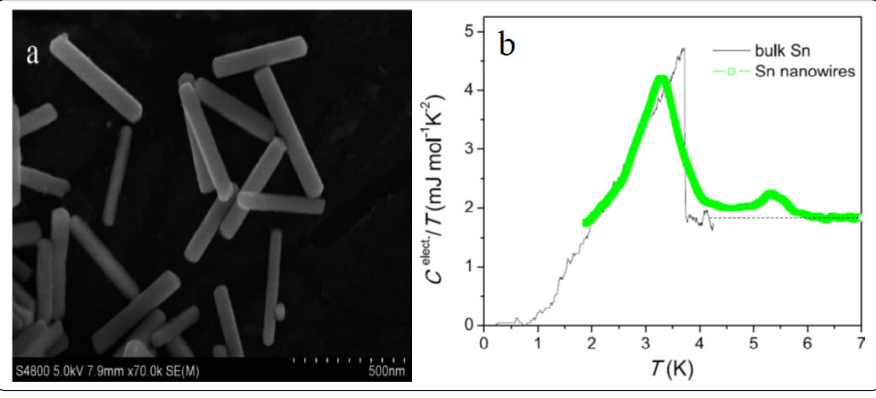

Figure 14 (a). SEM image of Sn nanowires. (b)The electronic heat capacity Celectr/T at the superconducting transition of Sn nanowires (open squares) as a function of temperature in zero field.

\section{Bi nanowires}

It is well-known that bulk rhombohedral $\mathrm{Bi}$ at ambient pressure is not a superconductor down to 50mk [89]. Rece nt studies on this "old" material have uncovered a number of new phenomena $[68,90]$. Rhombohedral Bi nanowires have been observed to become superconducting due to topo logical nature of the surface state of crystalline $\mathrm{Bi}$ in confined geometries [91-93].

Granular Bi nanowires with well-defined rhombohedral grains of $\sim 10 \mathrm{~nm}$ diameter, fabricated by electrochemically depositing $\mathrm{Bi}$ into porous polycarbonate membranes at ambient pressure [47], were superconducting with two transition temperatures, Tc, of 7.2 and $8.3 \mathrm{~K}$. These Tc values coincide with Tc values of the highpressure phases $\mathrm{Bi}-\mathrm{III}$ and $\mathrm{Bi}-\mathrm{V}$, respectively. The authors suggested that it could be due to the high-pressure phases existing in the 
grain boundaries.The sharp voltage steps were also observed in the V-I curves which were very similar to those found in quasione-dimensional superconducting nanowires.
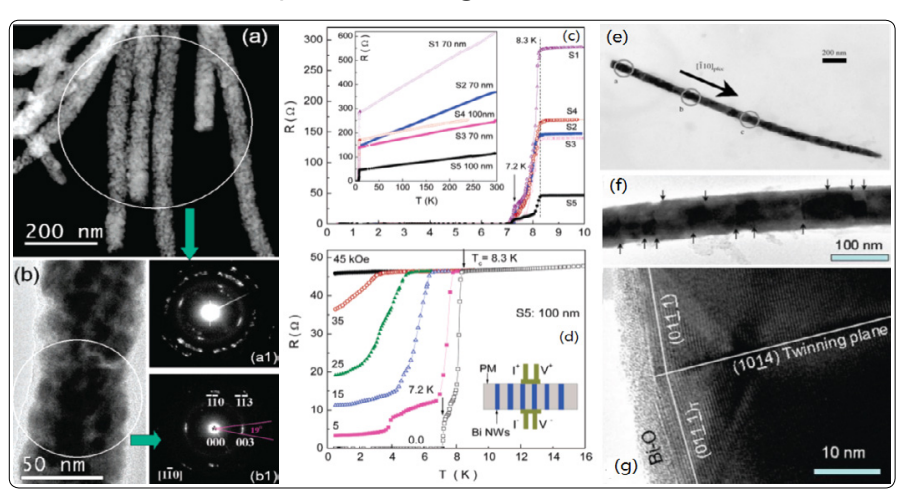

Figure 15 (a) and (b)The mophology of granular Bi nanowires. (c) Resistance $(R)$ versus temperatures $(T)$ of several superconducting granular Bi nanowires in low-temperature range. The inset is the $\mathrm{R}-\mathrm{T}$ curvesbin the temperature range of $0.45-300 \mathrm{~K}$ under zero magnetic field(H). (d) R-T curves of S5 measured at different $\mathrm{H}$ in the low $T$ range; the inset is the schematic arrangement of the transport measurement. (e)-(g)

Ye et al [94] reported experimental evidence for superconductivitybelow $0.64 \mathrm{~K}$ in electrochemically fabricated single-crystal Bi nanowires with a rhombohedral lattice structure of bulk $\mathrm{Bi}$ at ambient $\mathrm{p}$ ressure. As shown in Figure 15e there were some spots along the nanowire. Tian et al [95] fabricated bamboo-like morphology Bi nanowires with $79 \mathrm{~nm}$ in diameter. The high resolution TEM image (Figure $15 \mathrm{~g}$ ) taken near the "bamboo" stripe indicated that the stripes shown in Figure $15 f$ are actually small-angle twinning boundaries with a twinning plane of (1014), which was perpendicular to the growth direction of the wire. The log R-log T plot of (1014) twined Bi nanowires showed a shoulder around $0.67 \mathrm{~K}$ which was likely related to the same superconducting phase observed by Ye et al. This would suggest the $0.67 \mathrm{~K}$ superconducting phase shown in this paperand results of Ye could have its origin of superconductivity from the twinning planes. The observation of superconductivity below $1.3 \mathrm{~K}$ was associated with the interface between the surface oxide and the inner core of the Bi wire.

To further explores the transport properties of individual single-crystalline Bi nanowires, Tian et al. synthesized ultrathin $\mathrm{Bi}$ nanowires of 20 and $32 \mathrm{~nm}$ diameters(Figure 16a and b). Compared with $72 \mathrm{~nm}$ Bi wire,the superconducting transition at $1.3 \mathrm{~K}$ was seen in both 20 and $32 \mathrm{~nm}$ cylindrical nanowires, but the $0.67 \mathrm{~K}$ phase observed in $72 \mathrm{~nm}$ wire did not appear (shown in Figure 16c). However, their superconducting critical magnetic fields decreased with their diameter, which is the opposite of the expected behavior for thin superconducting wires. Quasiperiodic oscillations of magnetoresistance were observed in perpendicular fields but were not seen in the parallel orientation. This result is also consistent with the obse rvation of superconductivity of $\mathrm{Bi}$ confined in an opal host without surface oxidation [96]. The superconductivity in cylindrical nanowires or particles is most likely related to the surface curvature-induced stress in the nanowires or nanoparticles. Such stress will give rise to considerable distortion or disordering in the surface states of the cylindrical nanowires.
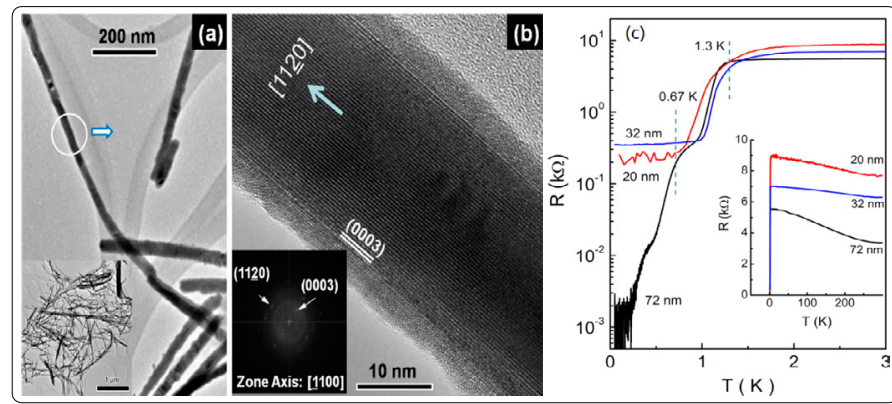

Figure 16 (a). Low magnification image of the wires; (b) high resolution image showing single crystalline morphology with a [1120] growth direction. (c) R-T plot at $\mathrm{H}=0$ Oe for 20, 32, and 72 $\mathrm{nm}$ diameter $\mathrm{Bi}$ nanowires in the low $\mathrm{T}$ range, measured with a standard 4-probe technique by using FIB deposited Pt electrodes. The inset shows the data between 0.1 and $300 \mathrm{~K}$.

\section{Zn nanowires}

Bulk zinc $(\mathrm{Zn})$ is a conventional type I superconductor(S) with a transition temperature at $0.85 \mathrm{~K}$ and a criticalmagnetic field of $50 \mathrm{Oe}$. Its superconducting cohere ncelength in a bulk crystalline sample, $\xi 0$, at $\mathrm{T}=0 \mathrm{~K}$ is foundto be as large as 2.0 $\mathrm{mm}$ [97], which is almost an order larger than those of the other materialsmentioned above. Therefore, one-dimensional (1D) effectsin Zn nanowires are expected to be more apparent comparedto other wires of the same diameter.

Zinc nanowires with diametersbetween 40 and $100 \mathrm{~nm}$ were fabricated by electrodepositing $\mathrm{Zn}$ in to commercially available polycarbonate (PC) or anodic aluminium oxide (AAO) membranes. [98], and study was conducted on the transport properties of the $\mathrm{Zn}$ nanowires.
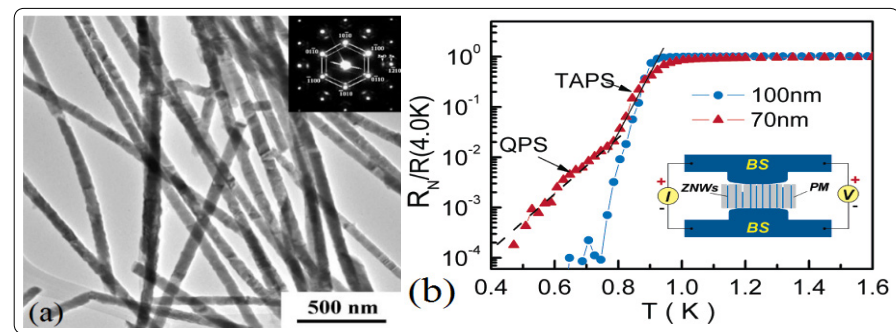

Figure 17 (a) TEM image of single-crystal Znnanowires, inset is the select area electron diffraction patterns (b) Normalized resistance vs temperature of 70- and 100-nm single-crystal Zn nanowires with a length of $6 \mu \mathrm{m}$.

Figure 17b showed the normalized resistance (RN/R (4.0K)) versus temperature $(\mathrm{T})$ of 70 - and 100-nm single-crystal Zn nanowires with a length of $6 \mu \mathrm{m}$, measured at a dc excitation current of $10 \mathrm{nA}$. The onset temperature, Tc, of both nanowires was found to be around $0.93 \mathrm{~K}$,

slightly higher than the Tc of bulk Zn $(0.84 \mathrm{~K})$. However, the

superconducting transition of $100 \mathrm{~nm}$ wires was sharp and the resistance drops to zero below $0.75 \mathrm{~K}(\Delta \mathrm{T} \approx 0.17 \mathrm{~K})$. In contrast, the transition of $70 \mathrm{~nm}$ wires broadened and showed a residual resistance extending down to $0.55 \mathrm{~K}$. Two distinct linear sections were found. The broadening of the transition near Tc was interpreted as a consequence of a thermally activated phase slip (TAPS) process [134] [135] in a 1D system. The second low-temperature exponential residual resistance that extends over a wide range far below Tcis probably from the quantum phase-slip process suggested first by Giordano. The result with 70-nm Zn wires is also consistent with Tian's previous observations of 20- and 40-nm single-crystal Sn nanowires; the only difference is that the resistive tail caused by the QPS process in $\mathrm{Zn}$ nanowires appears in relatively larger diameter wires. 
Kurtz et al. probed the thermodynamics of $Z n$ nanowires filling porous anodic alumina membranes (AAMs) with diameters of 230 and 23nm [99]. The critical temperature in zero field, def ined as the midpoint of the jump, for both samples was higher than in bulk ( $T_{c}$ bulk $\left.=0.85 \mathrm{~K}[100]\right): 0.89$ and $0.94 \mathrm{~K}$ for the small-pore and large-pore samples, respectively. The enhancement of the transition temperature may be due to local disorder though it is unclear why the larger wires are more affected. The height and breadth of the transition of the samples were plotted in Figure 18. The specific heat jump of bulk $\mathrm{Zn}$ crystal was slightly sharper than that of $\mathrm{Zn}$ nanowires. The spread in wire diameters in each sample and the dependence of the transition temperature on wire diameter can account for much of this width. The more rounded peak of the large-pore sample would thus be consistent with its larger spread in wire diameter. The height and breadth of the specific heat jump were virtually unaffected by the severe confinement. Evidently, the suppression of superconducting order by thermodynamic fluctuations was much more sensitively detected by electrical transport, since a zero-resistance state depends on long-range phase coherence along the entire length of the sample. Specific heat, on the other hand, probes more local superconducting order, which is apparently still quite robust even in $23 \mathrm{~nm}$ diameter nanowires that are nearly 7 times smaller than the coherence length.

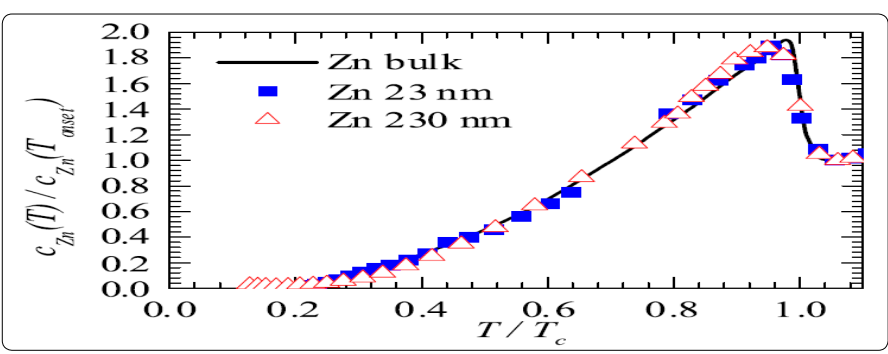

Figure 18. Normalized specific heat versus normalized temperature. The solid curve is bulk $\mathrm{Zn}$ data.

When a superconducting nanowire is connected to two strongly superconducting bulk electrodes, the total combined system is exp ected to be superconducting below the Tc of the superconducting nanowire and the electrodes [101-102]. The superconductivity of the wire is expected to become more robust through its coupling with the superconducting reservoirs. It was observed in Figure 19a-c that single-crystal Zn nanowires of diameter $30-250 \mathrm{~nm}$ and length $6 \mu \mathrm{m}$, using an in situ template-based electrochemical method [103], did not show any signature of superconductivity when they were in contact with $\mathrm{Au}$ electrodes, while $\mathrm{Zn}$ nanowires were superconducting at $\mathrm{Tc}(\mathrm{Sn})$ when they were in contact with $\mathrm{Sn}$ electrodes. And were superconducting at $\mathrm{Tc}(\mathrm{Pb})$ when they were in contact with $\mathrm{Pb}$ electrodes. Liu et al. concluded that when in contact with electrodes having a higher Tc, superconductivity was induced in these NWs at the Tc of the electrodes.

The results of Tian are contrary to this expectation in a system consisting of superconducting zinc nanowires (ZNWs) sandwiched between two bulk superconductors (BSs) of differe nt materials ( $\mathrm{Sn}$ and $\mathrm{In}$ ), which showed the strong effect of their superconducting environment [4]. It was found that the superconductivity of $\mathrm{Zn}$ nanowires of $40 \mathrm{~nm}$ diameter was suppressed either completely or partially by the superconducting electrodes. When the electrodes were driven into their normal state by a magnetic field, the nanowires switched back to their superconducting state. The phenomenon was not seen in wires with diameters equal to or thicker than $70 \mathrm{~nm}$, as shown in Figure 19d and e. However, when $\mathrm{Zn}$ nanowires of $40 \mathrm{~nm}$ in diameter and two micrometers in length sandwiched between $\mathrm{Pb}$ electrodes, the antiproximity effect (APE) found in this system was subtle, showing up primarily in significantly reducing the critical current of the $\mathrm{Zn}$ nanowires when the $\mathrm{Pb}$ electrodes were in a superconducting state. The critical current was independent of the magnetic field until it was increased to a characteristic value $\mathrm{Hd}^{d}$ near 0.6 kOe when the critical current showed a rapid enhancement and reaches a maximum value at the critical field of $\mathrm{Pb}$ electrodes at $0.94 \mathrm{kOe}$. The overall features of the R-T curves as shown in Figure $19 \mathrm{f}$ and $\mathrm{g}$ resembled those found in the 70 $\mathrm{nm}$ diameter ZNWs with bulk $\mathrm{Sn}$ or In electrodes where the ZNWs and electrodes both show superconductivity at their respective Tc.

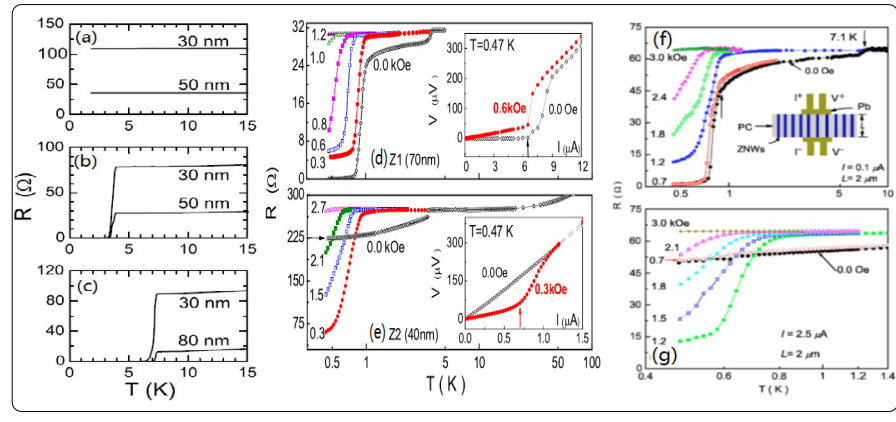

Figure 19 .R-T curves of (a)-(c) Zn NWs with Au (top), Sn (middle), or Pb (lower) electrodes; (d) and (e) Zn NWs (d=40 nm, L=6 $\mu \mathrm{m}$ ) with $\mathrm{Sn}$ electrodes under different magnetic fields. The insets show the $\mathrm{V}-\mathrm{I}$ curves at $\mathrm{H}=0: 0$ and $0.3 \mathrm{kOe}$, respectively. ( $\mathrm{f}$ ) and $(\mathrm{g}) \mathrm{ZNWs}$ $(\mathrm{d}=40 \mathrm{~nm}, \mathrm{~L}=2 \mu \mathrm{m})$ with $\mathrm{Pb}$ electrodes under excitation current, $\mathrm{I}=0.1 \mu \mathrm{A}$ and $\mathrm{I}=2.5 \mu \mathrm{A}$.

Fu et al [104] developed a theory for a superconducting nanowire coupled to various environments. This theory considered the boundary effect and phase-slip interaction in the presence of dissipative environment and suggested that the APE is due to the dissipation at the boundary between the ZNWs and the electrodes. When the nanowire of finite length is placed into a dissipation free environment, the ends of the wire can be mapped onto two parallel boundary lines that can screen the vortex-antivortexinteraction of the superconducting order parameter and destroys the superconducting phase even at $\mathrm{T}=0 \mathrm{~K}$. When the ends of the wire are coupled to a dissipative environment (such as normal metal electrodes), the screening becomes incomplete. As a result, for large dissipation the superconducting phase of the wire is stabilized. However, this model does not address the observation that the "strength" of APE is dependent on the materials of the superconducting electrode materials. Moreover, the APE has been observed only in zinc nanowires. 


\section{Al nanowires}

A convenient material to study the phenomena associated with QPS mechanismis aluminium. Its bulk critical temperature $T_{C}$ bulk 1.2 $K$ is relatively low, hence, the QPS rate should be comparatively high enabling pronounced manifestation ofthe effect. An additional useful feature of aluminium is its peculiar size dependenceof $T_{C}$. Although the origin of this effect remains unclear, an increase of $T_{C}$ withreduction of the characteristic dimension of aluminium structures (wire diameteror fil $\mathrm{m}$ th ickness) is a well-known experimental fact and can be taken as granted [105].

Zgirski et al $[5,106-107]$ showed that the application of low energy ( 1 keV) $\mathrm{Ar}+$ ion sputtering allows one to progressively and non destructively reduce dimensions of Al nanowireswidth down to $10 \mathrm{~nm}$. The electron mean free pathin such structures was estimated to be about or larger than the specimen thicknessand, therefore, such nanowires can be considered to be in the clean limitfor the transverse (confined) electron motion. An evolution of the shape of $R(T)$ dependencies in ul trathin and homogeneous aluminium nanowires as a function of their effective diameter was observed [5]. It was found (as shown in Figure 20) that for effective diameters $\sqrt{ } \sigma \geq 15 \mathrm{~nm}$ the $R(T)$ dependencies can be described by the model of thermally activated phase slips. For thinner wires the width of $R(T)$ transition is dramatically wider, and the resistance may not tend to zero at $T \rightarrow 0$. They associated this phenomenon with manifestation of quantum phase fluctuations (quantum phase slips). The systematic shift of the mean critical temperature Tc to higher values with reduction of the wire cross section. This is a well-known experimental fact: critical temperature of superconducting films (and wires) often differs from the corresponding bulk value. For aluminium the Tc always increases with the decrease of the minimum characteristic dimension.When the wire diameter was further reduced [106], deviations from the TAPS behaviour became obvious (Figure 20b). Fits to the TAPSmodel failed to provide any reasonable quantitative agreement with experiment for diameter values below $\sigma^{1 / 2} \leq 20 \mathrm{~nm}$ even if one hypothetically assumes the existence of unrealistically narrow constrictions not observed by SPM. The broadening of the $R(T)$ dependencies in aluminium nanowiresat $T<T_{C}$ bulk can hardly be ascribed to geometrical imperfections, such asconstrictions. On the contrary, the broadened $R(T)$ curves of the thinnest $\mathrm{Al}$ samples(Figure 20b) can be nicely fit with the QPS model. One can conclude that the most natural interpretation of the results [34] can be associated with quantum fluctuations.
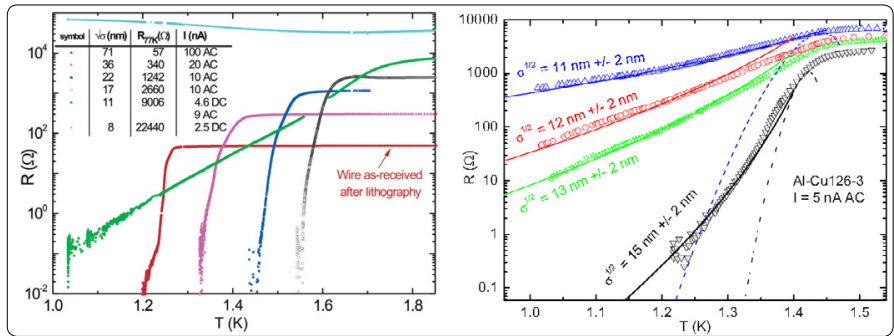

Figure $20(a) . R(T)$ curves for the same wire of length $L=10 \mu m$ after several sputtering sessions. (b) $R(T)$ curves for the thinnest samples obtained by progressive diameter reduction for thesame aluminium nanowire with length $L=10 \mu \mathrm{m}$. The TAPS model fitting is shown with dashedlines for 11 and $15 \mathrm{~nm}$ samples. Fits using QPS mechanism are shown by solid color lines
The ob served broad $R(T)$ dependencies in long $A l$ nanowires (up to $100 \mu \mathrm{m}$ )were associated with QPS (Figure 21) [6]. Although rather scarce experimental datamake quantitative conclusions on the QPS mechanism difficult, the experiments on long Al nanowires have a clear message: (a) the width of the experimental $R(T)$ transitions is much larger than predicted by TAPS model, (b) no correlationsbetween the total normal state wire resistance $R_{N}$ (compared to the quantum resistance unit $\mathrm{Rq}=6.45 \mathrm{k} \Omega$ ) and superconductivity in such wires was found. Altomare et al [37] fabricated Al nanowires with $8 \mathrm{~nm}$ wide using thermally evaporating aluminium onto a narrow molecular-beam epitaxy (MBE) grown $\mathrm{InP}$ ridge. Their results indicated that at higher $\mathrm{T}(\mathrm{T} / \mathrm{Tc}$ 20.7) the contribution from QTPS is negligible compared to TAPS, at low $T(T / T c \leq 0.5)$ TAPS is expected to be exponentially suppressed and QTPS should dominate. This expectation was confirmed by the fitswhich yielded a negligible RTAPS compared to RQTPS, as shown in Figure 22.
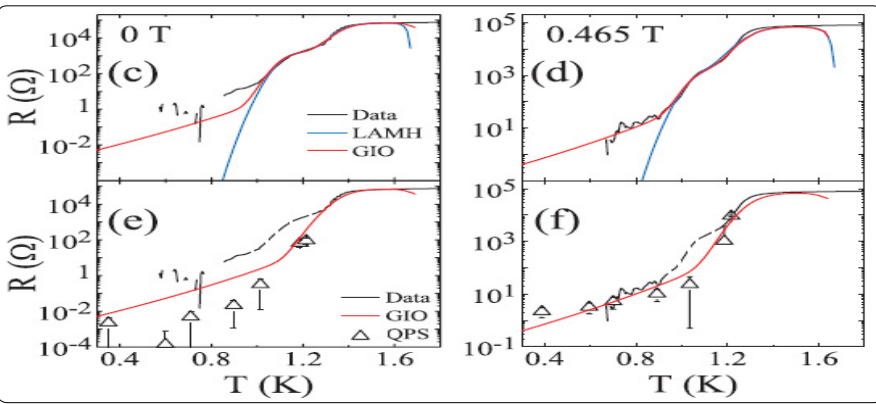

Figure 21. Experimental $R(T)$ dependence for $A$ I nanowires (symbols and black lines).

Solid colorlines stand for fits using TAPS (blue) and QPS (red) contributions

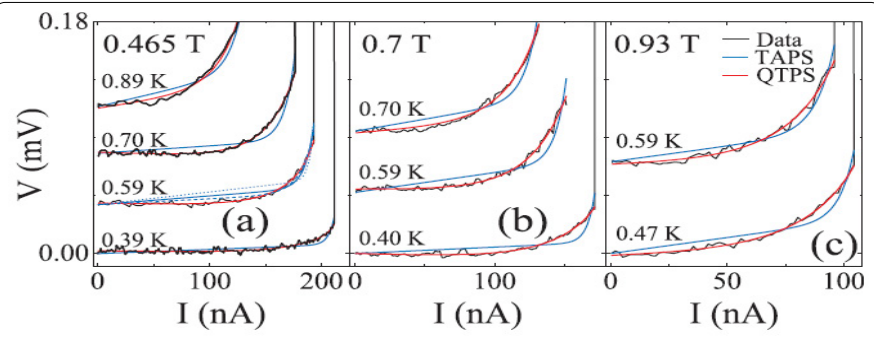

Figure 22. The nonlinear I- $V$ curves, at $T / T c \leq 0.5$ were refitted with $V$ $=\mathrm{V}_{\text {QTPS }}+\mathrm{V}_{\mathrm{S}}$ and $\mathrm{V}=\mathrm{V}_{\text {TAPS }}+\mathrm{V}_{\mathrm{S}}$.(a)-(c) The data (black), QTPS (red), and TAPS (blue) fits were shown after subtracting the linear background.

Croitoru et al [108] investigated the influence of the quantum size resonances on the critical magnetic field and the critical supercurrent in Al cylinder nanowire with diameter $D=3-15 \mathrm{~nm}$ and length $L=5 \mu \mathrm{m} \lambda_{F}$ based on a self-consistent numerical solution of the Bogoliubov-de Gennes equations. The formation of single-electron sub bands as a consequence of quantum confinement strongly influenced the superconducting-to-normal phase transition driven by a magnetic field. According to the Ginzburg-Landau theory this transition is of second order for mesoscopic wires, while being of first order in bulk type I superconductors. However, the situation changes significantly for narrower wires. As shown in Figure 23 , in a parallel magnetic field and/or in the presence of a supercurrent the transition from the superconducting to 
the normal phase occurred as a cascade of discontinuous jumps in the superconducting order parameter for diameters $\mathrm{D}<10 \sim 15 \mathrm{~nm}$ at $\mathrm{T}=0$. The critical magnetic field exhibited quantum-size oscillations with pronounced resonant enhancements as a function of the wire radius.
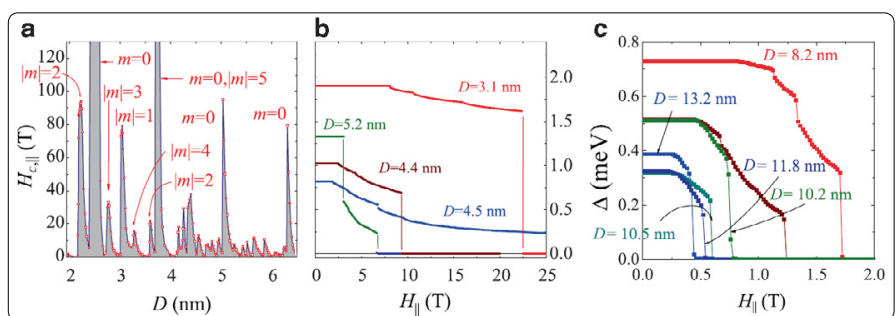

Figure 23 (a) Critical parallel magnetic field $\mathrm{H}_{c, \|}$ vs. the nanowire diameter $D$ and (b) spatially averaged order parameter as function of $\mathrm{H}_{\|}$for the resonant diameters $\mathrm{D}=3.1,4.4,4.5$, and $5.2 \mathrm{~nm}$. (c) Spatially averaged order parameter vs. $\mathrm{H}$ for the resonant diameters $\mathrm{D}=8.2,10.2,10.3,10.5,11.8$, and $13.2 \mathrm{~nm}$.

Figure 24a showed the calculated critical current density as a function of the nanowire diameter. This result showed that quantum-size effects play a very important role in the sizedependent increase of the critical current. Real samples exhibit inevitable cross-section fluctuations that will smooth those quantum-size oscillations in the critical current. Figure 24b displayed the critical superfluid velocity vscas a function of the wire diameter. This quantity defined as the velocity at which complete destruction of the condensate occurs exhibits the same trend.
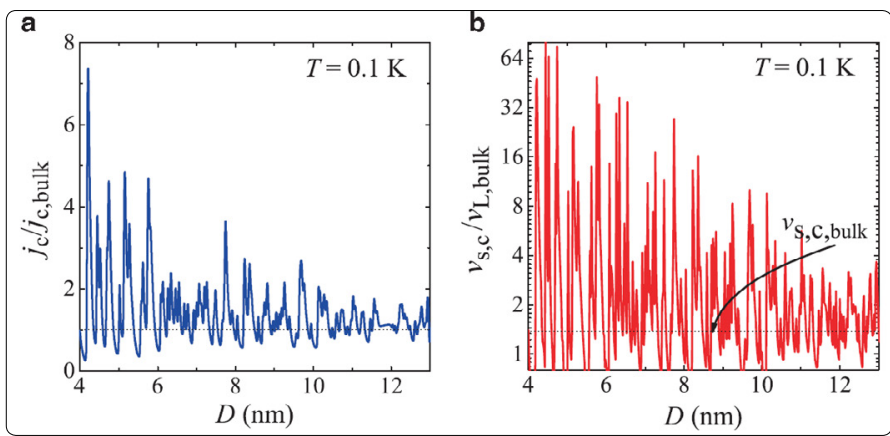

Figure 24 (a) Critical current and (b) critical superfluid velocity vs. the nanowire diameter calculated for $T=0.1 \mathrm{~K}$

\section{W nanowires}

Focused ion beams (FIB) technique offers the means to rapidly develop nanoscale devices in a single processing step in situ without the demand of time-consuming mask fabrication or extensive prior sample preparation. Tungsten is one of the most commonly used materials in FIB.

Córdoba [109] dealed with the growth of superconducting W NWs and W ultranarrow NWs grown by FIBID, with lateral size of by FIBID with lateral size $\sim 200 \mathrm{~nm}$ and $\sim 50 \mathrm{~nm}$ respectively. The magnetic field dependence $R(B)$ of the resistance of the W ultranarrow NWs showed non-monotonic $\mathrm{R}(\mathrm{B})$ behaviour and re-entrant zero-resistance state at lower temperature. At $B<B_{V}=1.61 \mathrm{~s}\left(\varphi_{0} / \mathrm{w}^{2}\right)$, the dissipation was caused bythe 'external' vortices crossing the wire. Every vortex saw a dome-like potential barrier with the maximum at the strip center and the height linearly decreasing with B.At $B=$ $B_{V}$, a single row of equilibrium vortices settled in the middle of the wire. Thus, at $B>B_{v}$ the dissipation was governed by the escape of these vortices from the strip across the barriers created by edge superconductivity. At $B \approx B_{V}$, the newly established edge barriers were nearly zero. As they also vanish at $B=B_{c 3}=1.695 B_{c 2}$, where $B c 2=\varphi_{0} / 2 \pi \xi_{d}{ }^{2}(T)$, where superconductivity was fully suppressed, these barriers must achieved their maximal value at some field between $B_{V}$ and $B_{c 3}$, giving rise to the $\mathrm{N}$-shape of $\mathrm{R}(\mathrm{B})$ with the maximum at $B_{V} \cdot I n$ addition to the re-entrance of the dissipation-free state observed in superconducting ultranarrow NWs a, the fundamental differences detected from structures of different lateral width were $\mathrm{Jc}$ and $\mathrm{B}_{\mathrm{c} 2,2 \mathrm{~K}}$. The critical current density without external magnetic field in superconducting $W$ ultranarrow NWs was one order of magnitude higher than the values obtained in both microwires and nanowires. In a bulk material, as W nanowires and microwires may be considered, the critical current density is governed by the pinningforces. However, if the dimensions of the structures are reduced to values close to $\xi$, superconductivity is preserved at the edges of the structures, so high current density is needed to be applied to overcome the energy barrier [110-112]. However, the upper critical field in the superconducting $\mathrm{W}$ ultranarrow NWs was at least a factor of 1.5 lower than the results obtained in $\mathrm{W}$ nanowires and microwires. This behaviour would be given by the energy dissipation mechanism, which holds exclusively in this kind of ultranarrow nanostructures in which width or thickness are of the same order as the coherence length.

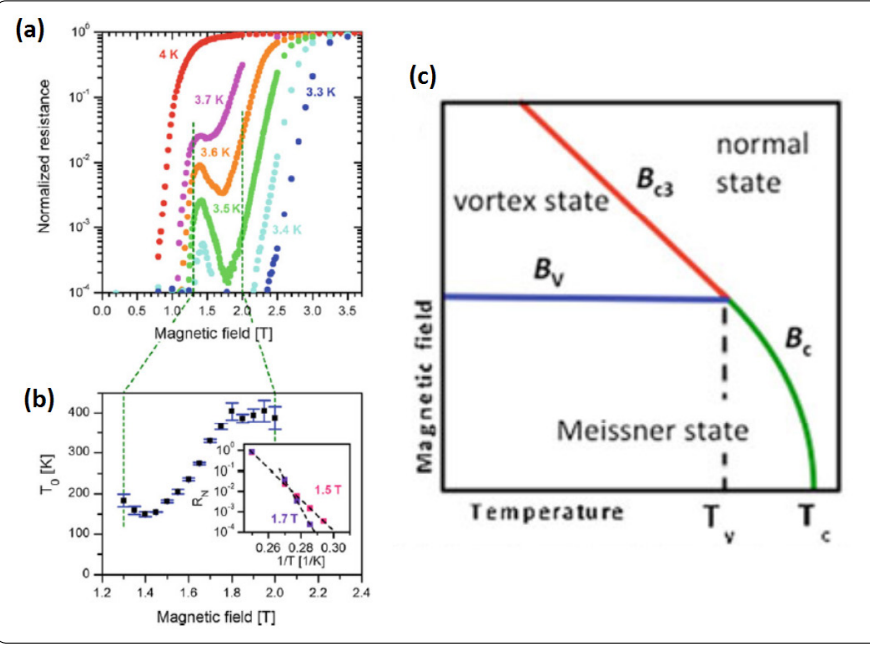

Figure 25 (a) The magnetic field dependences of the resistance of the W-based nanowire measured at different temperatures. (b) The variation of the activation energy $T_{0}$ with the magnetic field in the region of the formation of the re-entrant dissipation-free state. Inset Representative Arrhenius plot.(c) Sketch of the phase diagram obtained within the framework of the Ginzburg-Landau description of the superconducting wire with $\mathrm{w} \geq \xi_{\mathrm{d}}$.

Using the technique of FIB-CVD, Li et al [113] have grown ultra-thin and ultra-narrow tungsten lateral nanowires with width and thickness comparable to the phase coherence length (छ) of the bulk material. Temperature-dependent electrical measurements showed that the wires were conducting and had a superconducting transition with a transition temperature (Tc) about $5.1 \mathrm{~K}$. Resistance vs. temperature measurements revealed that (Figure 26) with decreasing cross-sectional area, the wires display an increasingly broad superconducting transition. A residual 
resistive tail extending down to the low-temperature region was found only for the thinnest tungsten nanowire, which is $10 \mathrm{~nm}$ thick and $19 \mathrm{~nm}$ wide. The logarithm of the residual resistance of this wire appeared as two linear sections as a function of temperature, one within $300 \mathrm{mK}$ below Tc and the other extending down to the lowest measuring temperature of $4.26 \mathrm{~K}$. Such features have previously been identified with phase slip processes as discussed before.

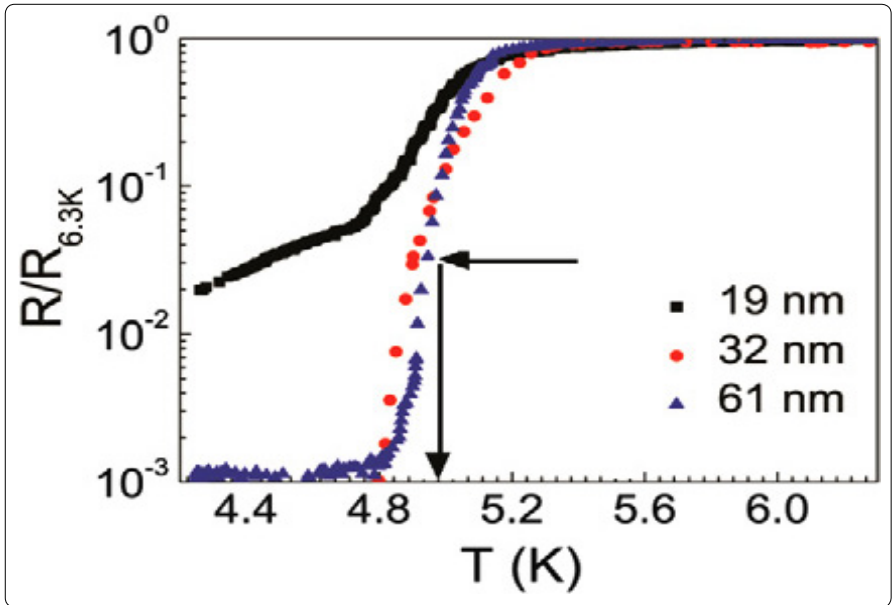

Figure 26. Normalized resistances vs temperature of the 19, 32 and $61 \mathrm{~nm}$ wide nanowires with normalized resistance in the log plot, showing distinct transition characteristics.

\section{Conclusion}

Remarkable progresses in quasi-one-dimensional superconducting single crystal nanostructures fabrication have been made in the past few years. Various techniques were employed, including directional deposition onto step edges, sputter deposition onto suspended carbon nanotube (CNT), electrodeposition into porous membranes, etch thinning of thick wires, superlattice nanowire pattern transfer (SNAP) method, chemical vapour deposition method, and solution-phase method. The superconducting single crystal nanowires are suitable for the establishment of the quantitative agreement between the theory and experiment. The key conclusions of these investigations can be summarized as follows. Fluctuations play an important role in high-quality metallic superconductors with nanoscale dimensions. In relatively thick samples, the finite width of experimental $R(T)$ transition can be qualitatively understood within the formalism of thermally activated phase slips (TAPS). Upon reduction of the wire diameter, the QPS rate increases drastically. If the wire diameter is decreased further (diameters $\leq 10 \mathrm{~nm}$ ), QPS proliferate causing a sharp crossover from a superconducting to a normal behaviour. The critical magnetic field exhibits quantum-size oscillations with pronounced resonant enhancements in thinner nanowires. However, the origin of the $T_{C}$ size dependence phenomenon is not clear yet. The thinner single crystalline niobium and aluminium nanowires have a lower $\mathrm{Tc}$; the thinner single crystalline tin nanowires have higher $T_{C}$ may be because of surface electron-phonon scattering effects; single crystal lead wires show higher Tc than that of polycrystalline ones, but no size dependence is observed.

\section{Acknowledgements}

This work was financially supported by the National Natural Science Foundation of China (No. 51271215) and the Innovative Exploratory Grant of Hong Kong University of Science and Technology (No. IEG14EG02PG).

\section{References}

1. Herzog AV, Xiong P, Sharifi F, Dynes RC. Observation of a Discontinuous Transition from Strong to Weak Localization in 1D Granular Metal Wires. Phys. Rev. Lett. 1996; 76: 668. doi: 10.1103/PhysRevLett.76.668

2. Camarota B, Parage F, Delsing P, Buisson O. Experimental Evidence of One-Dimensional Plasma Modes in Superconducting Thin Wires. Phys. Rev. Lett. 2001; 86: 480. doi: 10.1103/PhysRevLett.86.480

3. Vodolazov DY, Peeters FM, Piraux L, Mátéfi T S, Michotte S. CurrentVoltage Characteristics of Quasi-One-Dimensional Superconductors: An S -Shaped Curve in the Constant Voltage Regime. Phys. Rev. Lett. 2003; 91: 15700.

4. Tian M, Kumar N, Xu S, Wang J, Kurtz JS, Chan MHC. Suppression of Superconductivity in Zinc Nanowires by Bulk Superconductors. Physical Review Letters. 2005; 95: 076802.

5. Zgirski M, Riikonen KP, Touboltsev V, Arutyunov K. Size Dependent Breakdown of Superconductivity in Ultranarrow Nanowires. Nano Lett. 2005; 5: 1029-1033.

6. Fabio A, Chang AM, Michael RM, Yuguang H, Charles WT. Evidence for Macroscopic Quantum Tunneling of Phase Slips in Long OneDimensional Superconducting Al Wires. Physical Review Letters. 2006; (97): 017001. doi: 10.1103/PhysRevLett.97.017001.

7. Rogachev A, Bollinger AT, Bezryadin A. Influence of High Magnetic Fields on the Superconducting Transition of One-Dimensional $\mathrm{Nb}$ and MoGe Nanowires .Phys. Rev. Lett. 2005, 94: 017004.

8. Lukens JE, Warburton RJ, Webb WW. Onset of Quantized Thermal Fluctuations in "One Dimensional" Superconductors. Phys. Rev. Lett. 1970; 25: 1180-84.

9. Little WA. Decay of Persistent Currents in Small Superconductors. Phys. Rev. 1967; 156: 396.

10. Lau CN, Markovic N, Bockrath M, Bezryadin A, Tinkham M. Quantum Phase Slips in Superconducting Nanowires. Phys. Rev. Lett. 2001; 87: 217003.

11. Giordano N. Phys. Evidence for Macroscopic Quantum Tunnelling in One-Dimensional Superconductors. Rev. Lett. 1988; 61: 2137-140.

12. Sharifi F, Herzog AV, Dynes RC. Crossover from two to one dimension in insitu grown wires of Pb. Phys. Rev. Lett. 1993; 71: 428-31.

13. Boogaard GR, Verbruggen AH, Belzig W, Klapwijk TM. Resistance of superconducting nanowires connected to normal-metal leads. Phys. Rev. B. 2004; 69: 220503. doi: 10.1103/PhysRevB.69.220503

14. Blonder GE, Tinkham M, Klapwijk TM. Transition from metallic to tunnelling regimes in superconducting micro constrictions: Excess current, charge imbalance, and supercurrent conversion. Phys. Rev. B. 1982; 25: 4515. doi: 10.1103/PhysRevB.25.4515

15. Bezryadin A, Lau CN, Tinkham M. Quantum suppression of superconductivity in ultrathin nanowires. Nature (London). 2000; 404: 971-974.

16. Remeika M, Bezryadin A. Sub-10 nanometre fabrication: Molecular templating, electron-beam sculpting and crystallization of metallic nanowires. Nanotechnology. 2005; 16(8): 1172.

17. Maki K, Fulde P. Equivalence of Different Pair-Breaking Mechanisms in Superconductors. Phys. Rev. 1965; 140: A1586. doi: 10.1103/PhysRev.140. A1586

18. Johansson A, Sambandamurthy G, Shahar D, Jacobson N, Tenne R. Nanowire acting as a superconducting quantum interference device. Phys. Rev. Lett. 2005; 95(11): 116805-116805. 
19. Xiong $\mathrm{P}$, Herzog AV, Dynes RC. Negative Magneto resistance in Homogeneous Amorphous Superconducting Wires. Phys. Rev. Lett. 1997; 78(5): 927-930.

20. Herzog AV, Xiong P, Dynes RC. Magneto resistance oscillations in granular Sn wires near the superconductor-insulator transition. Phys. Rev. B.1998; 58(21): 14199-14202.

21. Yi G, Schwarzacher W. Single crystal superconductor nanowires by electrodeposition. Appl. Phys. Lett. 1999; 74(12): 1746-1748. doi: $10.1063 / 1.123675$

22. Michotte $\mathrm{S}$, Mátéfi TS, Piraux L. Current-voltage characteristics of $\mathrm{Pb}$ and Sn granular superconducting nanowires. Appl. Phys. Lett. 2003; 82: 41194121.

23. Mingliang $T$, Jinguo $W$, James $S K$, et al. Dissipation in quasi-onedimensional superconducting single-crystal Sn nanowires. Phys. Rev. B. 2005; 71: 104521.

24. Rogachev A, Bezryadin A. Superconducting properties of polycrystalline $\mathrm{Nb}$ nanowires templated by carbon nanotubes. Appl. Phys. Lett. 2003; 83: $512-514$.

25. Salvato $M$, Cirillo $C$, Fittipaldi $R$, et al. Transport properties in aggregates of $\mathrm{Nb}$ nanowires templated by carbon nanotube films. Carbon. 2016; 105: 544-550.

26. Lin $\mathrm{YH}$, Nelson J, Goldman AM. Superconductivity of very thin films: the superconductor-insulator transition. Phys. C. 2015; 514:130-141. doi: 10.1016/j.physc.2015.01.005

27. Kim H, Jamali S, Rogachev A. Superconductor-insulator transition in long MoGe nanowires. Phys. Rev. Lett. 2012; 109(2): 0270021-0270025. doi: 10.1103/PhysRevLett.109.027002

28. Bollinger AT, Rogachev A, Remeika M, Bezryadin A. Effect of morphology on the superconductor-insulator transition in one-dimensional nanowires. Phys. Rev. B. 2004; 69(18): 1805031-1805034.

29. Bollinger AT, Dinsmore RC, Rogachev A, Bezryadin A. Determination of the superconductor-insulator phase diagram for one-dimensional wires. Phys. Rev. Lett. 2008; 101(22): 2270031-2270034.

30. Dickey $J M$, Paskin A. Phonon spectrum in small particles and their implications for superconductivity. Phys. Rev. Lett. 1968; 21(20): 4411443. doi: 10.1103/PhysRevLett.21.1441

31. McMillan WL. Transition temperature of strong-coupled superconductors. Phys. Rev. 1968; 167 (2): 331-344. doi: 10.1103/ PhysRev.167.331

32. Eugene BG, Alexey VB, Alexander VK, Vladimir IM, Igor IK. Suppression of superconductivity in thin $\mathrm{Nb}$ nanowires fabricated in the vortex cores of superfluid helium. Physica C. 2015; 516: 44-49. doi: 10.1016/j. physc.2015.06.010

33. Zaikin AD, Golubev DS, Otterlo AV, Zimanyi GT. Phys. Rev. Lett. 1997; 78: 1552.

34. Zhao WW, Liu X, Chan MHW. Quantum Phase Slips in $6 \mathrm{~mm}$ Long Niobium Nanowire. Nano Lett. 2016; 16: 1173-1178.

35. Xu K, James RH. Controlled Fabrication and Electrical Properties of Long Quasi-One-Dimensional Superconducting Nanowire Arrays. Nano Letters. 2008; 8(1): 136-141.

36. Tinkham M, Lau CN, Markovic N. Resistance induced by quantum phaseslips in superconducting nanowires. Physica E. 2003; 18(1-3): 308-311. doi: 10.1016/S1386-9477(02)01033-0

37. Anderson PW. Physics of the Resonating Valence Bond (Pseudogap) State of the Doped Mott Insulator: Spin-Charge Locking. Phys Rev Lett. 2006; 96: 017001.

38. Wang J, Shi CT, Tian ML, et al. Proximity-induced superconductivity in nanowires: minigap state and differential magneto resistance oscillations. Phys. Rev. Lett. 2009; 102(24): 247003-247006. doi: 10.1103/ PhysRevLett.102.247003

39. Wang J, Ma XC, Lu L, et al. Anomalous magneto resistance oscillations and enhanced superconductivity in single-crystal $\mathrm{Pb}$ nanobelts. Appl. Phys. Lett. 2008; 92(23): 233119. doi: 10.1063/1.2945280
40. Wang J, Ma XC, Qi Y, et al. Negative magneto resistance in fractal $\mathrm{Pb}$ thin films on Si(111). Appl. Phys. Lett. 2007; 90(11): 113109.

41. Wang J, Ma XC, Qi Y, et al. Carrier transport properties of nanocrystalline Er3N@C80. J. Appl. Phys. 2009; 106: 034301. doi: 10.1063/1.4887796

42. Wang J, Jia JF, Ma XC, et al. Semiconductor-superconductor transition and magneto resistance terraces in an ultrathin superconducting $\mathrm{Pb}$ nanobridge. J. Vac. Sci. Technol. B. 2010; 28: 678. doi: 10.1116/1.3437016

43. Wang J, Ma XC, Ji SH, et al. Magneto resistance oscillations of ultrathin $\mathrm{Pb}$ bridges. Nano Res. 2009; 2(9): 671-677.

44. Wang J, Ma XC, Qi Y, et al. An unusual magneto resistance effect in the heterojunction structure of an ultrathin single-crystal $\mathrm{Pb}$ film on silicon substrate. Nanotechnology.2008; 19: 475708.

45. Guan $Z L$, Ning $Y X$, Song $C L$, et al. Sample-size dependence of the superconducting transition of ribbon-shaped $\mathrm{Pb}$ nanocrystals studied by scanning tunneling spectroscopy. Phys. Rev. B. 2010; 81: 054516. doi: 10.1103/PhysRevB.81.054516

46. Guo $Y$, Zhang $Y F$, Bao $X Y$, et al. Superconductivity modulated by quantum size effects. Science. 2004; 306: 1915.

47. Michotte S, Mótéfi TS, Piraux L. Investigation of superconducting properties of nanowires prepared by template synthesis. Supercond. Sci. Technol. 2003; 16: 557.

48. Tian ML, Kumar N, Wang JG, Xu SY, Chan MHW. Influence of a bulk superconducting environment on the superconductivity of onedimensional zinc nanowires. Phys. Rev. B.2006; 74: 014515

49. Liu HD, Ye ZX, Luo ZP, Rathnayaka KDD, Wu WH. Boundary effect on superconductivity in long single-crystal superconducting nanowires Original Research Article. Physica C. 2008; 468: 304.

50. Michotte S, Piraux L, Dubois S, Pailloux F, Stenuit G, Govaerts J. Superconducting properties of lead nanowires arrays. Physica C. 2002; 377(3): 267-276. doi: 10.1016/S0921-4534(01)01306-5

51. Tinkham M. Introduction to Superconductivity, seconded., McGraw-Hill, Inc., New York, 1996: 102-119.

52. Michotte S, Mátéfi TS, Piraux L. 1D-transport properties of single superconducting lead nanowires. Physica C. 2003; 391(4): 369-375. doi: 10.1016/S0921-4534(03)01086-4

53. Kramer L, Watts RJT. Theory of Dissipative Current-Carrying States in Superconducting Filaments. Phys. Rev. Lett. 1978; 40: 1041. doi: 10.1103/ PhysRevLett.40.1041

54. Michotte S, Mátéfi TS, Piraux L, Vodolazov DY, Peeters FM. Condition for the occurrence of phase slip centers in superconducting nanowires under applied current or voltage. Physical Review B. 2004; 69: 094512.

55. Mingquan $\mathrm{H}$, Chi Hw, Pok LT, et al. "Giant" Enhancement of the Upper Critical Field and Fluctuations above the Bulk Tc in Superconducting Lead Nanowire Arrays. ACS nano. 2013; 7: 4187-4193.

56. Charnaya EV, Tien C, Lin KJ, Wur CSK, Yu A. Superconductivity of Gallium in Various Confined Geometries. Phys. Rev. B. 1998; 58: 467-472.

57. Hagel J, Kelemen MT, Fischer G, et al. Superconductivity of a Crystalline Ga84-Cluster Compound. J. Low Temp. Phys. 2002; 129(3): 133-142.

58. Ohshima K, Fujita T. Enhanced Superconductivity in Layers of Ga Fine Particles.J. Phys. Soc. Jpn. 1986; 55: 2798-2802. doi: 10.1143/JPSJ.55.2798

59. Cohen RW, Abeles B. Superconductivity in Granular Aluminum Films. Phys. Rev. 1968; 168: 444-450. doi: 10.1103/PhysRev.168.444

60. Kresin VZ, Ovchinnikov YN. 'Giant' Strengthening of Superconducting Pairing in Metallic Nanoclusters: Large Enhancement of Tc and Potential for Room-Temperature Superconductivity. Sov. Phys. Usp. 2008; 51: 427-435.

61. Chandrasekhar BS. A Note on the Maximum Critical Field of High-Field Superconductors. Appl. Phys. Lett. 1962; 1: 7-8.

62. Fulde $P$, Ferrell RA. Superconductivity in a Strong Spin- Exchange Field Phys. Rev. 1964; 135: A550-A563. doi: 10.1103/PhysRev.135.A550

63. Larkin Al, Ovchinnikov YN. Quasiclassical Method in the Theory of Superconductivity. Sov. Phys. Jetp.1965; 20: 762-769. 
64. Jian W, Yi S, Mingliang T, Bangzhi L, Meenakshi S, Chan MHW. Superconductivity in single crystalline $\mathrm{Pb}$ nanowires contacted by normal metal electrodes. Physical review. B, Condensed matter. 2012; 86(3): 035439.

65. Wang J, Ma X, Lu L, et al. Anomalous magneto resistance oscillations and enhanced superconductivity in single-crystal $\mathrm{Pb}$ nanobelts. Appl. Phys. Lett. 2008; (92): 233119. doi: 10.1063/1.2945280

66. Tian M L, Wang J, Snyder J, et al. Synthesis and characterization of superconducting single-crystal Sn nanowires. Appl. Phys. Lett. 2003; 83: 1620-1622.

67. Li L, Checkelsky JG, Hor YS, et al. Phase transition of Dirac electrons in bismuth. Science. 2008; 321: 547-550.

68. Behnia K, Balicas L, Kopelevich Y. Signature of electron fractionalization in ultra quantum bismuth. Science. 2007; 317: 1729-1731.

69. Dubois S, Michel A, Eymery JP, Duvail JL, Piraux L, Mater J. Fabrication and properties of arrays of superconducting nanowires. Res. 1999; (14): 665-671. doi: 10.1557/JMR.1999.0091

70. Ning $\mathrm{W}$, Kong $\mathrm{FY}, \mathrm{Xi} \mathrm{CY}$, et al. Evidence of topological two-dimensional metallic surface states in thin bismuth nanoribbons. ACS Nano. 2014; 8 : $7506-7512$.

71. McCumber DE, Halperin BI. Time Scale of Intrinsic Resistive Fluctuations in Thin Superconducting Wires. Phys. Rev. B. 1970; (1): 1054. doi: 10.1103/PhysRevB.1.1054

72. Lubos J, Dimitrios G, Pantelis NT, et al. Carbon Nanotubes Encapsulating Superconducting Single-Crystalline Tin Nanowires. Nano letters. 2006; (6): $1131-1135$

73. Hsu YJ, Lu SY. Low temperature growth and dimension- dependent photoluminescence efficiency of semiconductor nanowires. J Phys. Chem. B. 2005; (109): 4398.

74. Han JE, Crespi VH. Asymmetry in negative differential resistance driven by electron-electron interactions in two-site molecular devices. Phys. Rev. B. 2004; (69): 214526. doi: 10.1063/1.1413499

75. Naugle DG, Baker JW, Allen RE. Evidence for a Surface-Phonon Contribution to Thin-Film Superconductivity: Depression of Tc by Noble-Gas Overlayers. Phys. Rev. B. 1973; (7): 3028. doi: 10.1103/ PhysRevB.7.3028

76. Lukens JE, Warburton RJ, Webb WW. Onset of Quantized Thermal Fluctuations in "One-Dimensional" Superconductors .Phys. Rev. Lett. 1970; 25: 1180. doi: 10.1103/PhysRevLett.25.1180

77. Newbower RS, Beasley MR, Tinkham M. Fluctuation Effects on the Superconducting Transition of Tin Whisker Crystals. Phys. Rev. B. 1972; 5: 864. doi: 10.1103/PhysRevB.5.864

78. Langer JS, Ambegaokar V. Intrinsic Resistive Transition in Narrow Superconducting Channels. Phys. Rev. 1967; 164: 498. doi: 10.1103/ PhysRev.164.498

79. Giordano N. Dissipation in a one-dimensional superconductor: evidence for macroscopic quantum tunneling. Phys. Rev. Lett. 1990; 41: 6350. doi: 10.1103/PhysRevB.41.6350

80. Ying $\mathrm{Z}$, Wong $\mathrm{CH}$, Shen J, et al. Dramatic enhancement of superconductivity in single crystalline nanowire arrays of Sn. Scientific Reports. 2016; 6: 32963.

81. Pracht US, Bachar N, Benfatto L, et al. Enhanced Cooper pairing versus suppressed phase coherence shaping the superconducting dome in coupled aluminum nanograins. Phys Rev. B. 2016; 93: 100503(R). doi: 10.1103/PhysRevB.93.100503

82. Mayoh J, García GAM. Strong enhancement of bulk superconductivity by engineered nanogranularity. Phys. Rev. B. 2014; 90: 134513.

83. Ginzbur VL. On surface superconductivity. Phys. Lett. 1964; 13: 101-102.

84. Wong $\mathrm{CH}$, Lortz R. Edge effect and significant increase of the superconducting transition onset temperature of $2 \mathrm{D}$ superconductors in flat and curved geometries. Physica C. 2016; 521-522: 50-54.
85. Hohenberg PC. Existence of long range order in one and two dimensions. Phys. Rev. 1967; 158: 383-386. doi: 10.1103/PhysRev.158.383

86. Mermin ND, Wagner $\mathrm{H}$. Absence of ferromagnetism or antiferromagnetism in one- or two-dimensional isotropic Heisenberg models. Phys. Rev. Lett. 1966; 17: 1133-1136. doi: 10.1103/ PhysRevLett.17.1133

87. Sekihara $T$, Masutomi $R$, Okamoto $T$. Magnetic-field-independent superconductivity of ultrathin $\mathrm{Pb}$ films on cleaved Gas surface. J. Phys.: Conf. Series. 2013; 456: 012034.

88. Yaji K, Ohtsubo Y, Hatta S, et al. Large Rashba spin splitting of a metallic surface-state band on a semiconductor surface. Nat. Comm. 2010; 1: $1-5$.

89. Overcash DR, Ratnam BA, Skove MJ, Stillwell EP. Persistent metallic behaviour of thin bismuth whiskers. Phys. Rev. Lett. 19; 44: 1348. doi: 10.1103/PhysRevLett.44.1348

90. Wells JW, Dil JH, Meier F, et al. Nondegenerate metallic states on Bi(114): a one-dimensional topological metal. Phys. Rev. Lett. 2009; 102: 096802.

91. Nikolaeva A, Gitsu D, Konopko L, Graf MJ, Huber TE. Quantum interference of surface states in bismuth nanowires probed by the Aharonov-Bohm oscillatory behaviour of the magnetoresistance. Phys. Rev. B. 2008; 77: 075332.

92. Zhu K, Wu L, Gong X, et al. The emergence of topologically protected surface states in epitaxial Bi(111).thin films. 2014.

93. Sabater C, Gosálbez MD, Fernández RJ, Rodrigo JGU, Palacios CJJ. Topologically Protected Quantum Transport in Locally Exfoliated Bismuth at Room Temperature. Phys. Rev. Lett. 2013; 110: 176802.

94. Zuxin $Y$, Hong Z, Haidong L, Wenhao W, Zhiping L. Evidence for superconductivity in single crystalline Bi nanowires. Physica B. 2008; 403 : 1529-1530. doi: 10.1016/j.physb.2007.10.180

95. Mingliang T, Jian W, Qi Z, Nitesh K, Thomas E. Mallouk, Chan MHW Superconductivity and Quantum Oscillations in Crystalline Bi Nanowire. Nano Lett. 2009; 9(9): 3196-3202.

96. Johnson RC, Nieshkoski MD, Disseler SM, Huber TE, Graf MJ. Superconductivity of Bi confined in an opal host. J. Low Temp. Phys. 2013; 170: 205-215.

97. Schulz $U$, Tidecks R. Dissipative state of superconducting zinc whiskers. J Low Temp. Phys. 1988; 71(1-2): 151-171.

98. Wang JG, Tian ML, Kumar N, Mallouk TE. Stretch-induced cell proliferation is mediated by FAK-MAPK pathway. Life Sci. 2005; (5): 1247-1253.

99. James SK, Robert RJ, Mingliang T, et al. Specific Heat of Superconducting Zn Nanowires. Phys. Rev. Lett. 2007; 98: 247001-4.

100. Seidel G, Keesom PH. Specific Heat of Gallium and Zinc in the Normal and Superconducting States. Phys. Rev. 1958; 112: 1083.

101. Cetas TC, Holste JC, Swenson CA. Heat Capacities from 1 to $30 \mathrm{~K}$ of $\mathrm{Zn}$, Cd, Sn, Bi, and Y. Phys. Rev. 1969; 182: 679.

102. Agassi $D$, Cullen JR. Current-phase relation in an intermediately coupled superconductor-superconductor junction. Phys Rev. B. 1996; 54: 10112."

103. Haidong L, Zuxin Y, Zhiping L, Rathnayaka KDD, Wenhao W. Boundary effect on superconductivity in long single-crystal superconducting nanowires. Physica C. 2008; 468: 304-309. doi: 10.1016/j.physc.2007.08.029

104. Fu HC, Seidel A, Clarke J, Lee DH. Stabilizing superconductivity in a nanowire by coupling to a dissipative environment. Phys Rev. Lett. 2006; 96: 157005

105. Shanenkon AA, Croitoru MD, Zgirski M, Peeters FM, Arutyunov K Nanoscale superconductivity: Nanowires and nanofilms. Phys. Rev. B. 2006; 74(5): 052502

106. Zgirski M, Riikonen KP, Touboltsev V, Arutyunov K. Ion beam shaping and downsizing of nanostructures. Phys. Rev. B. 2008; 77: 054508.

107. Zgirski M, Arutyunov KY. Resistive state of quasi-one-dimensional superconductors: Fluctuations vs. sample in homogeneity. Phys. Rev. $B$ 2007; 1(40): 160-162. 
108. Croitoru AAS, Peeters FM. Tuning the superconducting properties of nanomaterials. Complex Phenomena in Nanoscale Systems. 2009; 1-14.

109. Rosa CC. Superconducting Tungsten-Based Nanodeposits Grown by Focused Ion Beam Induced Deposition. 2013; 09: 95-132.

110. Tahara S, Anlage SM, Halbritter J, Eom CB, Fork DK, Geballe TH, Beasley MR. Critical currents, pinning, and edge barriers in narrow $\mathrm{YBa} 2 \mathrm{Cu} 3 \mathrm{O} 7-\mathrm{d}$ thin films. Phys. Rev. B. 1990; 41(16): 11203-11208.

111. Jones WA, Barnes PN, Mullins MJ, et al. Impact of edge-barrier pinning in superconducting thin films. Appl. Phys. Lett. 2010; 97(26): 262503. doi: $10.1063 / 1.3529945$
112. Elistratov AA, Vodolazov DY, Maksimov IL, Clem JR. Field-dependent critical current in type-II superconducting strips: combined effect of bulk pinning and geometrical edge barrier. Phys. Rev. B. 2002; 66(22): 220506.

113. Wuxia L, Fenton JC, Changzhi G, Warburton PA. Superconductivity of ultra-fine tungsten nanowires grown by focused-ion-beam directwriting. Microelectronic Engineering. 2011; 88: 2636-2638. 